\title{
Tension Distribution of Single Motor Units in Multitendoned Muscles: Comparison of a Homologous Digit Muscle in Cats and Monkeys
}

\author{
Marc H. Schieber, ${ }^{1}$ Michael Chua, ${ }^{2}$ Julien Petit, ${ }^{3}$ and Carlton C. Hunt ${ }^{4}$ \\ ${ }^{1}$ Departments of Neurology, Neurobiology and Anatomy, and Brain and Cognitive Science, Center for Visual Science, and \\ the Brain Injury Rehabilitation Program at St. Mary's Hospital, University of Rochester School of Medicine and Dentistry, \\ Rochester, New York, 14642; '2Department of Cell Biology and Physiology, Washington University School of Medicine, St. \\ Louis, Missouri 63110; 'Laboratoire de Physiologie de l'Action et de la Perception, College de France 75231, Paris \\ Cedex 05, France; and ${ }^{4}$ Department of Physiology, University of North Carolina, Chapel Hill, North Carolina 27514
}

To determine whether single motor units (MUs) in multitendoned muscles distribute tension to multiple tendons or instead focus tension selectively on a single tendon, we examined the distribution of tension generated by single MUs in the cat extensor digitorum lateralis (EDLat), and in its macaque homolog, the extensor digiti quarti et quinti (ED45). General properties of MUs (maximal tetanic tension, axonal conduction velocity, and twitch rise time) were similar in these muscles to those reported for other limb muscles in cats and monkeys. Most cat EDLat MUs were found to exert tension rather selectively on one of the three tendons of the muscle. Fast fatigable MUs were slightly but significantly more selective than fast fatigue-resistant and slow MUs. In contrast, and contrary to expectation, the macaque ED45 contained a lower proportion of MUs that exerted tension selectively on one of the two tendons of the muscle, and a higher proportion of relatively nonselective MUs. These findings suggest that the cat EDLat may consist of three functional subdivisions, each acting preferentially on a different tendon, whereas the macaque ED45 is more likely to function as a single multitendoned muscle.

Key words: compartment; distribution; force; functional; macaque; motor unit; multitendoned; muscle; neuromuscular; subdivision; tension
Humans, nonhuman primates, and to some extent cats and rats, make individuated movements of the digits of their hands or forepaws in which one or more digits move relatively independently of the movement or posture of the other digits (Gorska and Sybirska, 1980; Schieber, 1991; Soechting and Flanders, 1992; Whishaw and Gorny, 1994). This ability is all the more impressive when one considers how several of the major flexors and extensors of the digits differ from most other muscles: a single muscle belly gives rise to multiple tendons serving different digits. In humans, for example, flexor digitorum superficialis, flexor digitorum profundus, and extensor digitorum communis (EDC) each provide tendons to all four fingers. When these multitendoned muscles contract, they should move multiple digits simultaneously.

Recently, however, muscles in various mammalian species have been shown to consist of multiple functional subdivisions (Burke, 1995; English et al., 1993; Windhorst et al., 1989). Within such a muscle, each subdivision consists of a region of muscle fibers innervated by a separate branch of the muscle nerve (English and Letbetter, 1982; English and Weeks, 1984; Balice-Gordon and Thompson, 1988; English, 1990; Segal et al., 1991; Drake et al.,

\footnotetext{
Received July 10, 1996; revised Dec. 5, 1996; accepted Dec. 9, 1996.

This work was supported by National Institute of Neurological Disorders and Stroke Grants R01-NS27686 to M.H.S. and R01-NS27643 to C.C.H., and by a grant from the McDonnell Center for the Study of Higher Brain Function at Washington University. We thank David Becker for expert technical assistance, Tina Newman for assistance in data processing and figure preparation, and Marsha Hayles for editorial comments.

Correspondence should be addressed to Dr. Marc H. Schieber, University of Rochester Medical Center, Department of Neurology, 601 Elmwood Avenue, Box 673, Rochester, NY 14642.

Copyright (C) 1997 Society for Neuroscience 0270-6474/97/171734-14\$05.00/0
}

1993; Serlin and Schieber, 1993; Sanders, et al., 1994; Vanden Noven et al., 1994). In many of these muscles, the MUs of different subdivisions comprise functional sub-pools that the nervous system can activate differentially, exerting biomechanically different forces on the tendon of the muscle(s) (Herring et al., 1979; Pare et al., 1981; English, 1984; English and Weeks, 1987; Windhorst et al., 1989; Chanaud et al., 1991; Pratt et al., 1991; Pratt and Loeb, 1991; Schieber, 1993).

Human multitendoned extrinsic finger muscles often are assumed to contain such functional subdivisions, each devoted to a given finger. Electromyographic studies of extrinsic finger muscles in humans, for example, typically describe a single MU as optimally activated during movement of a particular finger (Schmied et al., 1993; Kilbreath and Gandevia, 1994). Studies examining the discharge of single MUs during individuated movements of each finger reveal, however, that a MU optimally activated during movement of a particular finger typically discharges during movements of adjacent fingers as well, albeit at a lower discharge frequency or at a higher recruitment threshold (Schieber, 1993; Kilbreath and Gandevia, 1994). Furthermore, most single MUs in the EDC of the cat have been shown to exert tension on all four digital tendons (Fritz et al., 1992). MUs in multitendoned muscles thus may not act exclusively on the tendon to only one finger.

Nevertheless, other multitendoned muscles might be organized into functional subdivisions that act preferentially on separate tendons. In particular, whereas the tendons of EDC are heavily interconnected in many species, the EDLat of the cat gives completely separate tendons to the three lateral digits, and its macaque homolog, ED45, gives two completely separate insertion tendons to digits 4 and 5 . We therefore examined the distribution 
of tension exerted by single cat EDLat MUs on the tendons to digits 3-5. Unlike the MUs of the EDC or extensor carpi ulnaris (ECU) of the cat (Fritz et al., 1992), EDLat MUs showed a high degree of selectivity for one of the three tendons of the muscle. The EDLat of the cat, therefore, might be segregated into three functional subdivisions, each of which exerts tension preferentially on one of the three tendons of the muscle. Although we expected a similar or even higher degree of tendon selectivity in MUs of the macaque ED45, we found instead that many ED45 MUs exerted roughly comparable tension on both tendons. The macaque ED45 more likely functions, therefore, as a single muscle that acts on digits 4 and 5 in parallel.

\section{MATERIALS AND METHODS}

All procedures for the care and use of both cats and monkeys complied with the U.S. Public Health Service Policy on Humane Care and Use of Laboratory Animals, followed the P.H.S. Guide for the Care and Use of Laboratory Animals, and were approved by the appropriate Institutional Animal Care and Use Committee. All nonsurvival procedures were performed under deep surgical anesthesia.

Cat preparation. Studies were performed on 5 female mongrel cats weighing 2-4 kg. Cats were premedicated intraperitoneally with 0.1 $\mathrm{mg} / \mathrm{kg}$ acepromazine, $0.04 \mathrm{mg} / \mathrm{kg}$ atropine, and $20 \mathrm{mg} / \mathrm{kg}$ ketamine; anesthetized with $20 \mathrm{mg} / \mathrm{kg}$ pentobarbital, i.p.; and thereafter received supplemental doses of pentobarbital i.v. to maintain deep surgical anesthesia, assessed by the absence of blink and pinna reflexes. Core body temperature was maintained at $38^{\circ} \mathrm{C}$ using a heating blanket controlled with a rectal temperature probe.

Once the cat was surgically anesthetized, the skin on the dorsal surface of either the right or left forelimb was opened with a single incision extending from just proximal to the elbow, down to the dorsum of the palm. Exposed tissues were kept moist with warmed Kreb's solution. The tendons of EDC and extensor carpi radialis were severed at the wrist; these muscles were dissected proximally and removed, exposing the distal radial nerve as it innervated the remaining distal forelimb extensors. The radial nerve then was dissected carefully into its component fascicles. Bipolar electrical stimulation $(50 \mu \mathrm{s}, 20 \mathrm{~Hz}, 10-100 \mathrm{~V}$ stimuli produced by a battery-powered optically isolated stimulator driven by an AMPI Master 8 stimulation timing unit, and delivered via a pair of hook electrodes spaced $3 \mathrm{~mm}$ apart) was used to determine whether each fascicle innervated EDLat, ECU, abductor pollicis longus, or extensor digiti 1 and 2. This determination was aided in some cases by placing a silk ligature around the tendon(s) of each muscle at the wrist. During stimulation of a given nerve fascicle, motion of the silk ligatures then could be observed easily, or the ligatures could be used to determine the presence or absence of twitch tension in each tendon. Nerve fascicles not contributing innervation to EDLat were cut and dissected for approximately $1.5 \mathrm{~cm}$ from the remainder of the radial nerve. Fascicles contributing innervation to both EDLat and another muscle were subdivided until all distal forelimb muscles innervated by the radial nerve, other than EDLat, had been denervated. Two separate fascicles innervating only EDLat typically remained. The radial nerve was then followed proximally, and branches to supinator, brachioradialis, and triceps were identified with stimulation and then cut. Via a separate incision on the medial aspect of the upper forelimb, or in some cases over pectoralis, the median and ulnar nerves were identified with electrical stimulation and cut.

The animal then was placed prone, the head was held with a maxillary clamp, and a bilateral laminectomy was performed, exposing the spinal cord and nerve roots from C6 through T3. The vertebral column was supported with a clamp on the spinous process of T4. The dura mater was divided longitudinally in the dorsal midline, and the free edge ipsilateral to the forelimb under study was gently retracted laterally, lifting the spinal nerve roots. The dorsal rootlets were cut from C6 through T2, exposing the ventral rootlets, which remained intact. Additional exposure of the ventral rootlets was obtained by gently retracting the spinal cord contralaterally with blunt glass nerve hooks. The spinal cord and nerve roots were protected in a bath of warm mineral oil.

The dissected forelimb was placed in a swimmer's crawl position, with the paw anterior, the arm and forearm in approximately the same horizontal plane as the shoulder, the elbow extended to roughly $120^{\circ}$, and the shoulder slightly protracted. The paw was held in a rigid clamp, and the distal radius and the olecranon process of the ulna were fixed separately with bone pins. The dorsal forelimb incision was reopened. A separate 5-0 silk suture then was placed in each of the three tendons of EDLat at the same proximodistal level near the wrist. (For brevity, we refer to the tendon to digit 3, 4, or 5 as $\mathrm{t} 3$, t4, or t5, respectively.) Each tendon was cut $5 \mathrm{~mm}$ distal to the suture. Loose connective tissue between the tendons was dissected proximally to the level of the muscle belly, maximizing the mechanical independence of the tendons. The belly of EDLat, its tendons, the innervating nerve fascicles, and the exposed distal radial nerve were protected in a bath of warm mineral oil.

The 5-0 silk suture in each EDLat tendon then was tied to a separate tension transducer (Load Cell BG-100 gm, Kulite Semiconductor Products, Inc.) mounted on a custom-made miniature rack and pinion that permitted adjustment of resting tendon length within a $10 \mathrm{~mm}$ range. All three transducers were positioned initially such that the sutured points in the three tendons were at the same proximodistal level as they had been in situ, thereby approximating the natural physiological length. The signal from each transducer was passed through a custom amplifier that permitted adjustment of baseline bias, and permitted gain switching such that a $0-10 \mathrm{~V}$ output represented either $0-10$ or $0-100 \mathrm{gm}-\mathrm{wt}$ tension. Each amplified signal was displayed on an analog oscilloscope and digitized at $2 \mathrm{kHz}$ through an Indec interface using custom CLab software run on a minicomputer (Compaq 20e). All nerve fascicles innervating EDLat then were stimulated simultaneously while the resting length of each tendon was adjusted to maximize twitch tension without prolongation of the twitch rise and fall times. When lengthening one tendon caused a decline in the twitch tension of another tendon, rest lengths were adjusted to optimize the simultaneous twitches in all three tendons. This procedure presumably minimized, on average, the possibility that muscle fibers acting on one tendon would be working at a different proportion of their physiological length than muscle fibers acting on the other tendons.

Data collection. To record axonal action potentials, the radial nerve fascicles innervating EDLat were placed on a hook electrode in the mineral oil bath, and an indifferent electrode was placed in nearby subcutaneous tissue. Action potentials in the nerve fascicles were amplified (Grass P511 or WPI DAM80 preamplifier), displayed continuously on an analog oscilloscope, and displayed on a Nicolet Explorer digital oscilloscope time-locked to stimulus pulses. A saline-filled glass micropipette electrode placed with a micromanipulator in the ventral rootlets was used to stimulate MUs. Ventral rootlets of the exposed spinal segments were systematically explored either rostrocaudally or caudorostrally. Stimuli were delivered between the micropipette (cathode) and a nearby subcutaneous return electrode (anode). As the electrode was advanced through a ventral rootlet, stimulus voltage was adjusted to evoke all-or-nothing action potentials plus tension responses from a single MU. Single MUs were identified during stimulation at $20 \mathrm{~Hz}$ as (1) unitary fiber action potentials in a nerve fascicle innervating EDLat, (2) occurring at a fixed latency after stimulation of a ventral rootlet, and (3) associated with measurable tension in at least one EDLat tendon. For each MU identified, the latency from stimulation to onset of the fiber action potential was measured on the Nicolet Explorer digital oscilloscope, and simultaneous tension recordings were made from the three tendons during isolated twitches elicited with single shocks at $<1 \mathrm{~Hz}$ (unpotentiated), and during $1 \mathrm{sec}$ tetanic stimulation at $20 \mathrm{~Hz}, 40 \mathrm{~Hz}$, and $150 \mathrm{~Hz}$ (Fig. 1). Data for off-line analyses were collected starting $250 \mathrm{~ms}$ before and ending $2000 \mathrm{~ms}$ after the onset of each stimulus, and stored to disk. In the first two cats, data files for single twitches were not stored.

After the exposed ventral rootlets had been explored for MUs innervating EDLat, the hook electrode again was used to stimulate the radial nerve fascicles supplying EDLat. Stimulation of whole muscle twitches, similar to those observed at the beginning of recording, confirmed that the overall contraction of the muscle had not deteriorated. The cat then was euthanized with a lethal injection of pentobarbital. The radial nerve, brachial plexus, and nerve roots were exposed, and the distance from the point of stimulation to the point of recording was measured.

Monkeys. Similar studies were performed on 4 female rhesus monkeys (Macaca mulatta, 4-9 kg) and 1 cynomologous monkey (Macaca fascicularis, $3 \mathrm{~kg}$ ). The cynomologous monkey was the first available for study. Twenty-two MUs were studied using radial nerve stimulation (see below). Because the properties of these MUs were indistinguishable from MUs studied in rhesus monkeys, and because previous anatomical studies have found no anatomical differences in ED45 among other Macaca species (Serlin and Schieber, 1993), the cynomologous MUs were pooled with rhesus MUs for analysis.

Monkeys were premedicated with $15 \mathrm{mg} / \mathrm{kg}$ ketamine and $0.04 \mathrm{mg} / \mathrm{kg}$ atropine, and then anesthetized with $25 \mathrm{mg} / \mathrm{kg}$ thiopental i.v., using 

$\mathrm{Tw}$
$20 \mathrm{~Hz}$
$40 \mathrm{~Hz}$
$150 \mathrm{~Hz}$

A
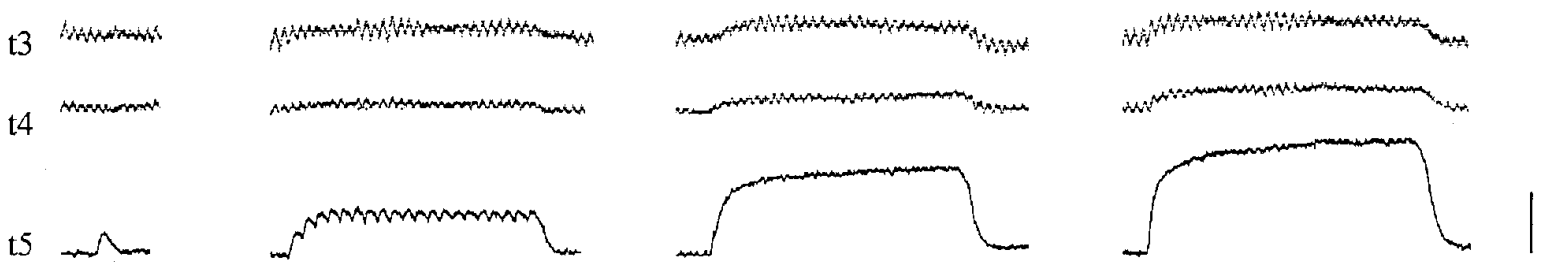

$\mathrm{B}$
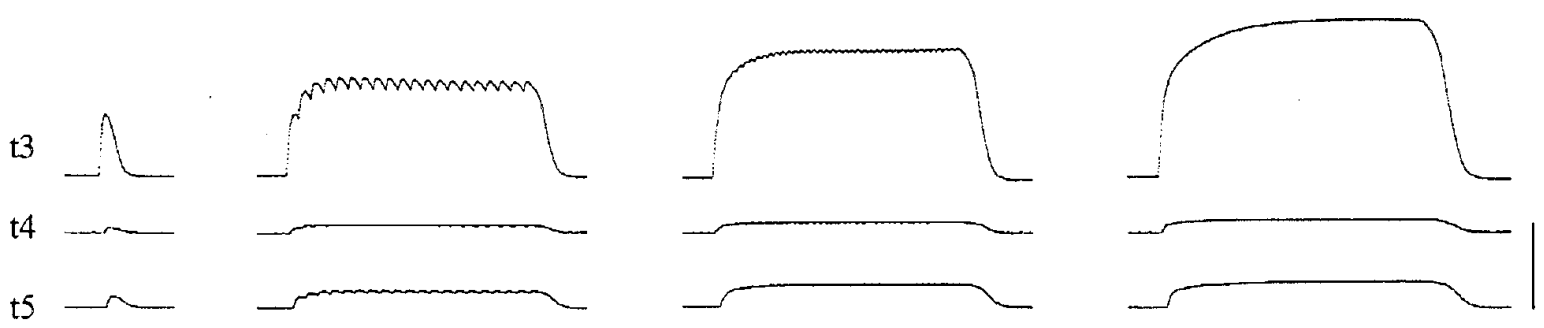

$\mathrm{t} 3$
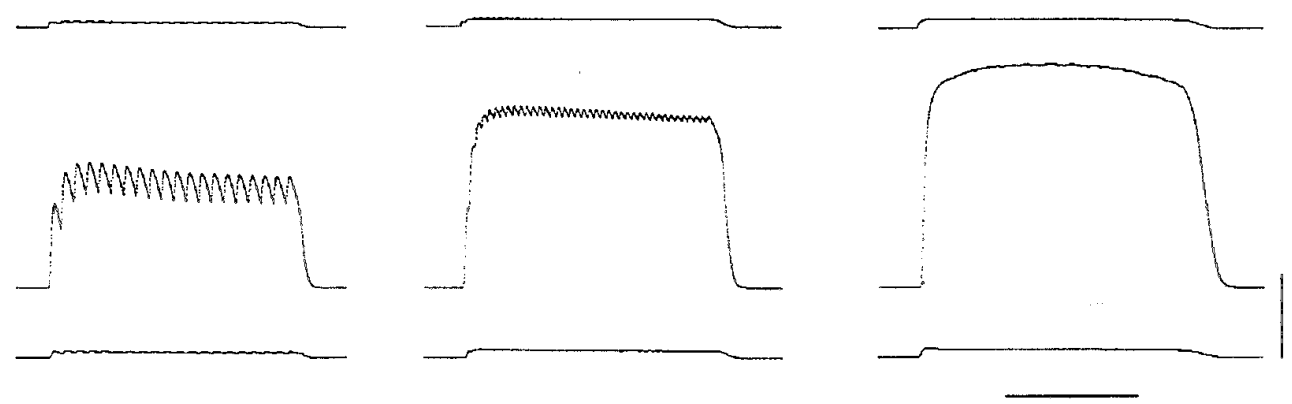

Figure 1. Three cat EDLat MUs. For each MU, simultaneous records of the tension on the tendon to digit $3(t 3)$, on the tendon to digit $4(t 4)$, and on the tendon to digit $5(t 5)$ are shown during single twitches and during tetanic stimulation at 20,40 , and $150 \mathrm{~Hz}$. $A$, The MU shown was classified as type $\mathrm{S}$, and exerted most of its tension on $\mathrm{t} 5$. B. The MU shown was classified as type FR, and exerted most of its tension on $\mathrm{t} 3$. $C$, The MU shown was classified as type FF, and exerted most of its tension on $\mathrm{t} 4$. Note the sag of the $\mathrm{t} 4$ tension of this MU after the first few stimuli of the $20 \mathrm{~Hz}$ tetanus. Such sag during $20 \mathrm{~Hz}$ tetani is characteristic of type FF MUs, and is not seen in type S or type FR MUs $(A$ or $B)$. All three MUs were from the same preparation. Horizontal calibration bar at bottom represents $0.5 \mathrm{sec}$; vertical calibration bars at right represent $0.2 \mathrm{gm}-\mathrm{wt}$ in $A, 0.5 \mathrm{gm}$-wt in $B$, and $20 \mathrm{gm}$-wt in $C$.

supplemental doses as needed to maintain deep surgical anesthesia, assessed as in cats. Monkeys were intubated and continuously monitored with EKG, pulse oximetry, end-tidal $\mathrm{CO}_{2}$, and a rectal temperature probe. Core body temperature was maintained at $37^{\circ} \mathrm{C}$ with heating blankets.

Preparation of the ED45, radial nerve, and spinal cord of the monkey was otherwise like that of the EDLat of the cat, with the exception that the radial nerve of the monkey was apparently much more subject than the that of the cat to development of conduction block. In three cases, contraction of ED45 could not be obtained by stimulation of the ventral rootlets after laminectomy, although such stimulation produced good contraction of proximal muscles, and stimulation of the dissected radial nerve fascicles produced good contraction of ED45. In two cases, we were able to stimulate single MUs in ED45 using glass micropipettes inserted into the radial nerve in the distal upper arm. But in the third case, even this portion of the radial nerve failed to conduct. Because inadvertent pressure on the already freed radial nerve during the final phases of preparation might have contributed to the block, for the last two monkeys we first cut the median and ulnar nerves through an upper arm incision, next performed the laminectomy, and then performed the forelimb dissection just before data collection.

Data analysis. Data files were reviewed off-line with custom minicomputer software that displayed the recorded tension waveforms graphically, and permitted time and amplitude measurements to be made with a mouse-controlled cursor. Further analyses were performed on a minicomputer using Microsoft Excel version 5.0.

For each MU, the peak tensions developed on each tendon during 4 single twitches and during tetani at 20,40 , and $150 \mathrm{~Hz}$ were measured. For each twitch, the contraction time was measured as the rise time from $10 \%$ to $90 \%$ of the peak amplitude on the tendon developing the greatest twitch tension. Using the rise time from only the tendon with the largest twitch avoided two problems. First, the smaller twitches sometimes were too close to the noise level for their rise times to be measured accurately. Second, if tension on the other tendons is transmitted to them passively, the rise time of their twitches may be prolonged by the viscous properties of the muscle, and therefore may fail to characterize the contracting muscle fibers accurately. The $10-90 \%$ rise time was averaged over the 4 twitches, and this average was used to characterize contraction time for 
A

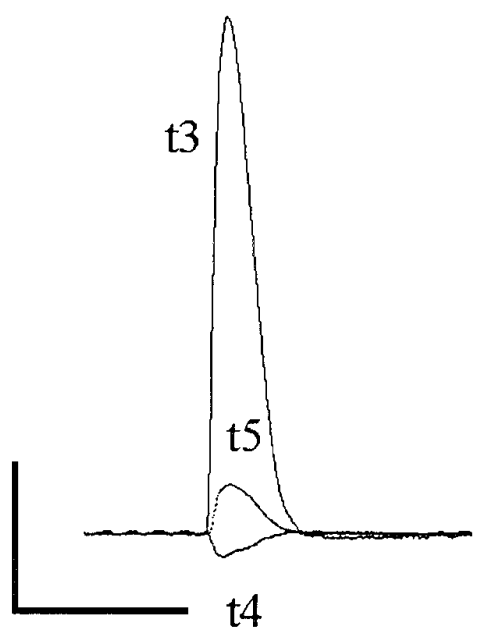

$\mathrm{B}$

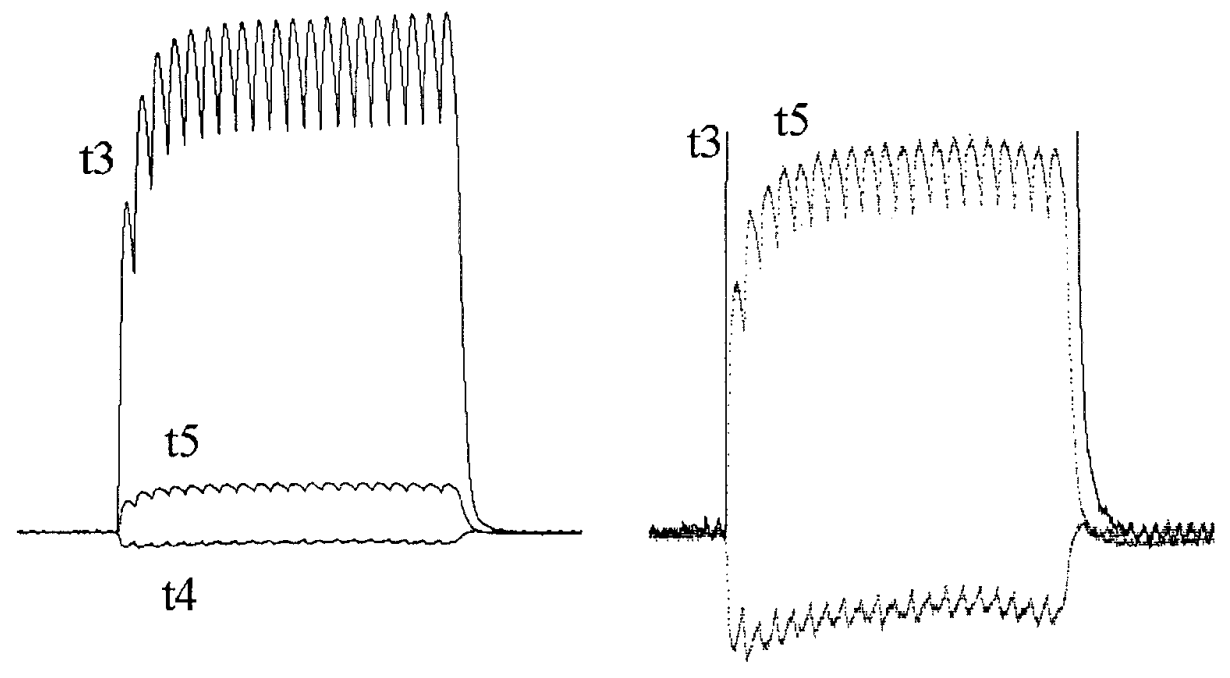

t4

Figure 2. Unloading of an EDLat tendon by a MU. Simultaneous tension records of EDLat's three tendons are shown during a single twitch $(A)$ and during tetanic stimulation at $20 \mathrm{~Hz}(B, C)$ of a single MU that unloaded the $\mathrm{t} 4$ tendon while exerting tension on the $\mathrm{t} 3$ and $\mathrm{t} 5$ tendons. In the single twitch $(A)$ and in the high gain record $(C)$, tension in the $t 4$ tendon can be seen to decrease with each increase in tension in the other two tendons. Horizontal calibration bar $=0.5 \mathrm{sec}$; vertical calibration bar $=1.51 \mathrm{gm}$-wt in $A, 2.36 \mathrm{gm}$-wt in $B, 0.30 \mathrm{gm}$-wt in $C$.

each MU. For any MUs lacking recorded single twitch data, we examined the $20 \mathrm{~Hz}$ tetanus record for distinct twitches. If distinct twitches were evident, the $10-90 \%$ rise time was measured from the first twitch of the $20 \mathrm{~Hz}$ tetanus. Accurate measurement of twitch rise time was precluded by a low signal-to-noise ratio for some of the very smallest MUs, and rise time data from these MUs was excluded from analyses. The conduction velocity of each MU was calculated by dividing the measured nerve conduction distance by the conduction latency of the MU.

MUs were classified into three physiological types-slow (S); fast fatigue-resistant (FR), and fast fatigable (FF)-by comparing the features of tension records from 20 versus $40 \mathrm{~Hz}$ tetani (Petit et al., 1990). As illustrated in Figure $1 A$, slow MUs developed slowly rising tension, without sag during the $20 \mathrm{~Hz}$ tetanus. Peak tensions were low during 20 $\mathrm{Hz}$ tetani, and little additional tension developed during $40 \mathrm{~Hz}$ tetani. Although individual twitches were only partially fused at $20 \mathrm{~Hz}$, the tetanus of type S units was fused at $40 \mathrm{~Hz}$. Type FR MUs (Fig. 1B) developed more quickly rising and higher peak tensions, without sag during unfused tetani at $20 \mathrm{~Hz}$. Peak tension was greater at $40 \mathrm{~Hz}$ than at $20 \mathrm{~Hz}$. Tetani at $20 \mathrm{~Hz}$ were less fused in type FR MUs than in type S MUs, and still were not totally fused at $40 \mathrm{~Hz}$. FF MUs (Fig. 1C) developed even more abruptly rising and still larger tensions, with appreciable sag during $20 \mathrm{~Hz}$ tetani. (Sag was the most important criterion for classifying a MU of intermediate size and twitch speed as FR vs FF.) Peak tension was greater at $40 \mathrm{~Hz}$ than at $20 \mathrm{~Hz}$. Tetani at both 20 and $40 \mathrm{~Hz}$ were even less fused in FF than in FR MUs. This qualitative method of classification produced groups of MUs the properties of which generally were consistent with those in other studies (see Results and Discussion below). Nevertheless, because each MU was assigned to one of the three physiological types based on our qualitative assessment of three properties (tension, tetanic fusion, and sag), and because we did not confirm these classifications with a fatigue test, some MUs may have been misclassified (Burke et al., 1973; Botterman et al., 1985; Petit et al., 1990; Fritz and Schmidt, 1992). Using the quantitative fatigue test, however, Petit et al. (1990) found that this qualitative method only occasionally misclassified a fast intermediate (FI) MU as type FF.

The maximal tension exerted on each tendon by a given MU was measured from the record of a $150 \mathrm{~Hz}$ tetanus, and the total tension for the MU was calculated as the sum of the tension exerted on each tendon. Tension during a $150 \mathrm{~Hz}$ tetanus was chosen to characterize the maximal output of each MU, recognizing that this stimulation frequency is well above the physiological range. Although the vast majority of MUs exerted measurable tension on all tendons, 13 cat EDLat MUs and 1 monkey ED45 MU unloaded some tendons while exerting tension on others (Fig. 2). For a given MU, unloading of the same tendon(s) typically was observed during single twitches, and during tetani at 20,40 , and $150 \mathrm{~Hz}$; the inverted rise time in unloaded tendons paralleled the rise time in loaded tendons. We therefore assume that these MUs, while loading one or two tendons, put tension on the aponeurosis of origin of the muscle sufficient to unload the other tendon(s) passively. Unloading was observed in at least one MU in each of the 5 cats, and unloading of each of the three tendons of the EDLat was observed at least once, making it unlikely that these unloading responses resulted from a generally inappropriate mechanical arrangement. Nevertheless, because such MUs would be unlikely to unload tendons during normal MU recruitment in vivo, for purposes of data analysis we assigned the tension in unloaded tendons a value of zero.

The maximal tension exerted on each tendon during a $150 \mathrm{~Hz}$ tetanus was used to calculate three indices quantifying the tendon selectivity of each MU. Two of these indices [output index (OPI) and divergence (DIV)] were originally developed by Fritz et al. (1992). Computation of all three indices is simplified by expressing the tension in each tendon as a fraction of the total tension exerted. If $T_{i}$ is the absolute tension in the $i$ th tendon of the muscle, then the fractional tension, $\tau_{i}$, is:

$$
\tau_{i}=T_{i} / \sum_{i=1}^{n} T_{i}
$$

where $n$ is the number of tendons.

The OPI quantifies the center of effort of the MU from -1 to +1 , with -1 representing all tension exerted on the most ulnar tendon, 0 representing tension distributed symmetrically about the center, and +1 representing all tension exerted on the most radial tendon.

The OPI is calculated as:

$$
O P I=\sum_{i=1}^{n} \tau_{i}^{*} w_{i}
$$


where $\tau_{i}$ is the fraction of the total tension exerted on the $i$ th tendon, $n$ is the number of tendons, and $w_{i}$ is a constant that provides a rankordered weighting of the tendons:

$$
w_{i}=(2 i-n-1) /(n-1) .
$$

The DIV quantifies the degree to which tension is focused on one tendon $(\mathrm{DIV}=0)$ versus being spread evenly over the tendons $(\mathrm{DIV}=1)$. The DIV is calculated as:

$$
D I V=s^{*} \sum_{i=1}^{n} \tau_{i}^{*}\left(O P I-w_{i}\right)^{2}
$$

where $s$ is a scaling factor that normalizes for the number of tendons:

$$
s=3^{*}(n-1) /(n+1) \text {. }
$$

The OPI and DIV are inter-related indices based on a radial-to-ulnar rank-ordered weighting of the tendons of a muscle. As such, they do not express the selectivity of a MU independent of which particular tendons receive what fractions of the total tension, assuming instead that tension will be distributed across the tendons with a single peak. The OPI thus can be ambiguous in that a value of 0 may indicate either that all the tension of the MU was exerted on the central tendon, or that tension was exerted symmetrically on either side of the central tendon. These two possibilities are distinguished by the DIV, which is 1 in the former case, and $<1$ in the latter, approaching 0 as tension becomes distributed evenly on all tendons. The DIV will exceed 1 , however, if the tension of the MU on the central tendon is less than that on the radial or ulnar tendons, reaching 1.5 for MUs that exert no tension on the central tendon(s) and equal tensions on the radial and ulnar tendons. This possibility apparently was not encountered by Fritz et al. (1992).

To express tendon selectivity of each MU independent of which particular tendons receive what fractions of the total tension, we therefore developed a third index (SEL) as follows. The fractional tensions of an ideally unselective MU that exerted equal tension on all of the tendons of a muscle would be $\tau_{u}=1 / n$, whereas the fractional tensions of an ideally selective MU that exerted tension on only one tendon, $\mathrm{t} 1$ for example, would be $\tau_{1}=1, \tau_{2}=0, \ldots \tau_{n}=0$. In an $n$-dimensional fractional tension space, the linear distance between these two points would be:

$$
d_{\max }=\sqrt{\left(1-\tau_{u}\right)^{2}+\sum_{i=2}^{n}\left(0-\tau_{u}\right)^{2}} .
$$

The linear distance between the point representing any other MU and the ideally unselective MU would be:

$$
d=\sqrt{\sum_{i=1}^{n}\left(\tau_{i}-\tau_{u}\right)^{2}} .
$$

The selectivity index for each MU is then calculated as:

$$
\mathrm{SEL}=d / d_{\max }
$$

SEL will vary from 0 for an ideally unselective MU to 1 for an ideally selective MU. Calculated from the fractional tensions of the MU, SEL is independent of the absolute magnitude of tension, allowing comparison of MUs of various size. SEL also is normalized for the number of tendons in the muscle, to permit comparison of MUs from muscles with different numbers of tendons. Unlike DIV, SEL is independent of which particular tendons receive what fractional portions of the tension of the MU. For example, DIV would be 0.9 for a hypothetical MU with fractional tensions $\tau_{1}=0.4, \tau_{2}=0.4$, and $\tau_{3}=0.2$; but DIV would be 1.2 for another hypothetical MU with fractional tensions $\tau_{1}=0.4, \tau_{2}=0.2$, and $\tau_{3}=0.4$. In contrast, SEL would be 0.2 for both of these hypothetical MUs.

Statistical comparisons of MU properties among physiological MU types were performed using one-way ANOVA. If significant variation was found, post hoc comparisons were made using two-tailed $t$ tests, assuming unequal variance, and applying Bonferonni correction for multiple tests. Because the derived indices (OPI, DIV, and SEL) are not normally distributed, statistical comparisons of these indices were made with the nonparametric, two-sample Kolmogorov-Smirnov (KS) test.

\section{RESULTS \\ Cat EDLat MUs}

We studied 169 cat EDLat MUs, from 22 to 49 in individual cats. These samples constitute roughly $10-20 \%$ of the EDLat motoneuron population in individual animals, shown to number 226-263 by retrograde labeling with horseradish peroxidase (Fritz et al., 1986). Consistent with these labeling studies, we found EDLat MUs over two consecutive spinal segments in each cat (either C7 to C8 or C8 to T1). Overall, 7 EDLat MUs were found in the $\mathrm{C} 7$ segment, 98 in $\mathrm{C} 8$, and 64 in $\mathrm{T} 1$.

Responses to tetanic stimulation at all three frequencies (20, 40, and $150 \mathrm{~Hz}$ ) were recorded from 161 of the 169 EDLat MUs. The total maximum tetanic tension of these 161 EDLat MUs, calculated as the sum of the tension in each of the 3 tendons during the $150 \mathrm{~Hz}$ tetanus, ranged from 0.05 to $69 \mathrm{gm}-w \mathrm{t}$. Conduction velocity, available for 134 of the 161 EDLat MUs, ranged from 47 to 94 $\mathrm{m} / \mathrm{sec}$. Twitch $10-90 \%$ rise time, available for 126 of the 161 EDLat MUs, ranged from 15 to $70 \mathrm{msec}$.

On the basis of comparison of the tension records of each MU from 20 and $40 \mathrm{~Hz}$ tetani, 36 were assigned to type $\mathrm{S}(22.4 \%), 73$ to FR $(45.3 \%)$, and 52 to FF $(32.3 \%)$. Table 1 gives descriptive statistics of the maximum tetanic tension, conduction velocity, and twitch rise time for each MU type in EDLat. Although the ranges of each of these three properties for the different MU types overlapped considerably, one-way ANOVA showed highly significant variation of each property in relation to MU type $(p<$ 0.0001). Post hoc comparisons of type $\mathrm{S}$ versus FR, $\mathrm{S}$ versus FF, and FR versus FF MUs also showed highly significant differences in each property between each pair of MU types $(p<0.0001)$, except that the conduction velocities of FR and FF MUs were significantly different only at $p<0.05$, and the twitch rise times of FR and FF MUs did not differ significantly.

Figure 3 shows the inter-relationships among tetanic tension, conduction velocity, and twitch rise time of EDLat MUs. Considering all MU types together, a significant positive correlation was found between the logarithm of total maximum tetanic tension and conduction velocity ( $r=0.744 ; p<0.0001$; Fig. $3 A$ ), and significant negative correlations were found between twitch rise time and conduction velocity $(r=0.529 ; P<0.0001$; Fig. $3 B)$ and between total tension and twitch rise time $(r=0.260 ; p<0.01$; Fig. $3 C$ ). As shown for a number of hindlimb muscles by EmonetDénand et al. (1988), the positive correlation between total tension and conduction velocity for EDLat MUs seemed to be a continuum among type S and FR MUs $(r=0.806 ; p<0.0001)$, whereas tension and conduction velocity were not significantly correlated for type FF units alone $(r=0.224 ; p>0.1)$.

\section{Distribution of tension on the three tendons of EDLat}

Figure 1 shows tension records from three different MUs during single twitches and during tetani at 20, 40, and $150 \mathrm{~Hz}$. These three MUs [one type S (Fig. $1 A$ ), one type FR (Fig. $1 B$ ), and one type FF (Fig. 1C)] each exerted tension rather selectively on one of the three different EDLat tendons. As all three of these MUs were studied in the same preparation, their selectivity is unlikely to be an artifact of the mechanical arrangement being slack on one or two tendons. The pattern of tension distribution of each MU across the three tendons remained relatively constant as stimulation frequency was varied, although the increase in tension at higher tetanus frequencies tended to be most marked in the tendon receiving the most tension (Fritz et al., 1992).

To examine how the population of EDLat MUs distributed tension across the tendons of the muscle, scatter plots were made 


\begin{tabular}{|c|c|c|c|c|}
\hline & $\mathrm{S}$ & FR & $\mathrm{FF}$ & All \\
\hline$N$ & 36 & 73 & 52 & 161 \\
\hline Percentage & 22.4 & 45.3 & 32.3 & 100 \\
\hline \multicolumn{5}{|c|}{ Maximum tetanic tension (gm-wt) } \\
\hline Mean $\pm \mathrm{SD}$ & $0.79 \pm 0.56$ & $11.9 \pm 14.2$ & $36.1 \pm 15.3$ & $17.2 \pm 18.9$ \\
\hline Range & $0.09-2.12$ & $0.05-66.5$ & $8.8-68.7$ & $0.05-68.7$ \\
\hline$n$ & 36 & 73 & 52 & 161 \\
\hline \multicolumn{5}{|c|}{ Conduction velocity $(\mathrm{m} / \mathrm{sec})$} \\
\hline Mean $\pm \mathrm{SD}$ & $56.7 \pm 6.8$ & $72.3 \pm 8.3$ & $76.3 \pm 8.0$ & $70.3 \pm 10.9$ \\
\hline Range & $47.3-73.1$ & $58.7-87.2$ & $55.5-94.5$ & $47.3-94.5$ \\
\hline$n$ & 30 & 53 & 51 & 134 \\
\hline \multicolumn{5}{|c|}{ Twitch $10-90 \%$ rise time (ms) } \\
\hline Mean \pm SD & $42.2 \pm 11.8$ & $30.0 \pm 5.6$ & $28.1 \pm 5.8$ & $31.4 \pm 8.8$ \\
\hline Range & $29.3-70.3$ & $15.6-46.9$ & $15.6-39.1$ & $15.6-70.3$ \\
\hline$n$ & 23 & 52 & 51 & 126 \\
\hline \multicolumn{5}{|l|}{ OPI } \\
\hline Mean $\pm \mathrm{SD}$ & $-0.014 \pm 0.663$ & $-0.050 \pm 0.587$ & $0.058 \pm 0.595$ & $-0.007 \pm 0.605$ \\
\hline Range & -1 to +1 & -1 to +0.976 & -0.878 to +0.943 & -1 to +1 \\
\hline$n$ & 36 & 73 & 52 & 161 \\
\hline \multicolumn{5}{|l|}{ DIV } \\
\hline Mean $\pm \mathrm{SD}$ & $0.628 \pm 0.440$ & $0.700 \pm 0.346$ & $0.383 \pm 0.247$ & $0.581 \pm 0.368$ \\
\hline Range & $0-1.22$ & $0-1.32$ & $0.043-1.11$ & $0-1.32$ \\
\hline$n$ & 36 & 73 & 52 & 161 \\
\hline \multicolumn{5}{|l|}{ SEL } \\
\hline Mean \pm SD & $0.632 \pm 0.288$ & $0.631 \pm 0.201$ & $0.784 \pm 0.136$ & $0.681 \pm 0.217$ \\
\hline Range & $0.160-1$ & $0.152-1$ & $0.387-0.956$ & $0.152-1$ \\
\hline$n$ & 36 & 73 & 52 & 161 \\
\hline
\end{tabular}

of the tension of each MU on a given tendon versus its tension on each of the other tendons during a $150 \mathrm{~Hz}$ tetanus. In Figure $4 A$, each MU is represented by a point in each of three plots. On the left, the tension of the MU on $\mathrm{t} 4$ is plotted versus its tension on $\mathrm{t} 3$; in the center, $\mathrm{t} 5$ tension is plotted versus $\mathrm{t} 3$ tension; and on the right, $\mathrm{t} 4$ tension is plotted versus $\mathrm{t} 5$ tension. Most FR and FF MUs are represented by points lying close to one axis but away from the origin, suggesting that tension was exerted rather selectively on the tendon corresponding to that axis. Only a few FR or FF MUs are represented by points between the axes, indicating that they exerted comparable tension on two tendons. Points representing smaller FR and S MUs form a cloud close to the origin in Figure $4 A$. These data, therefore, were replotted at an expanded scale in Figure $4 B$. Although a larger proportion of small MUs exerted comparable tension on two tendons, many small MUs also are represented by points lying close to one axis but away from the origin, again suggesting that they exerted tension rather selectively on the tendon corresponding to that axis. These plots, however, inadequately depict how each MU distributed its tension among all three of the tendons of EDLat simultaneously.

We therefore normalized the tension of each MU in each tendon as a fraction of the total tension of that MU (see Materials and Methods, Eq. 1). Because the sum of the fractional tensions for each MU is unity, in a three-dimensional scatter plot of fractional tensions, the points representing all
EDLat MUs will lie on the plane defined by $\tau_{3}+\tau_{4}+\tau_{5}=1$, where $\tau_{3}, \tau_{4}$, and $\tau_{5}$ denote the fraction of tension exerted by a MU on the tendons to digits 3, 4, and 5. (Note that the subscripts used here denote the digit on which tension would be exerted via the tendon, whereas in the calculation of OPI and DIV the ordinal tendon numbers 1,2 , and 3 must be used in place of digit numbers 3, 4, and 5, respectively.) Such a scatter plot is shown in Figure 5, where the axes have been rotated such that all points lie in the plane of the page. All points in this plot will fall within an equilateral triangle, the corners of which lie at unity along each axis. A MU that exerted all its tension on one tendon, and none on the other two, would be represented by a point at the appropriate corner. The triangle will be bounded by the lines (Fig. 5, fine dashed lines): $\tau_{3}+\tau_{4}=1, \tau_{5}$ $=0 ; \tau_{3}+\tau_{5}=1, \tau_{4}=0$; and $\tau_{5}+\tau_{4}=1, \tau_{3}=0$. A MU that distributed all its tension to two tendons, and none to the third, would be represented by a point on the appropriate edge of the triangle. A MU that distributed equal tension to all three tendons would be represented by a point in the center of the triangle, overlying the intersection of the three axes.

Figure 5 shows that the majority of EDLat MUs exerted tension rather selectively on one of the three tendons. Most points fell near a corner of the triangle. Points lying very close to a corner represent MUs that exerted almost all their tension on one tendon. Points less close to a corner represent MUs that exerted 

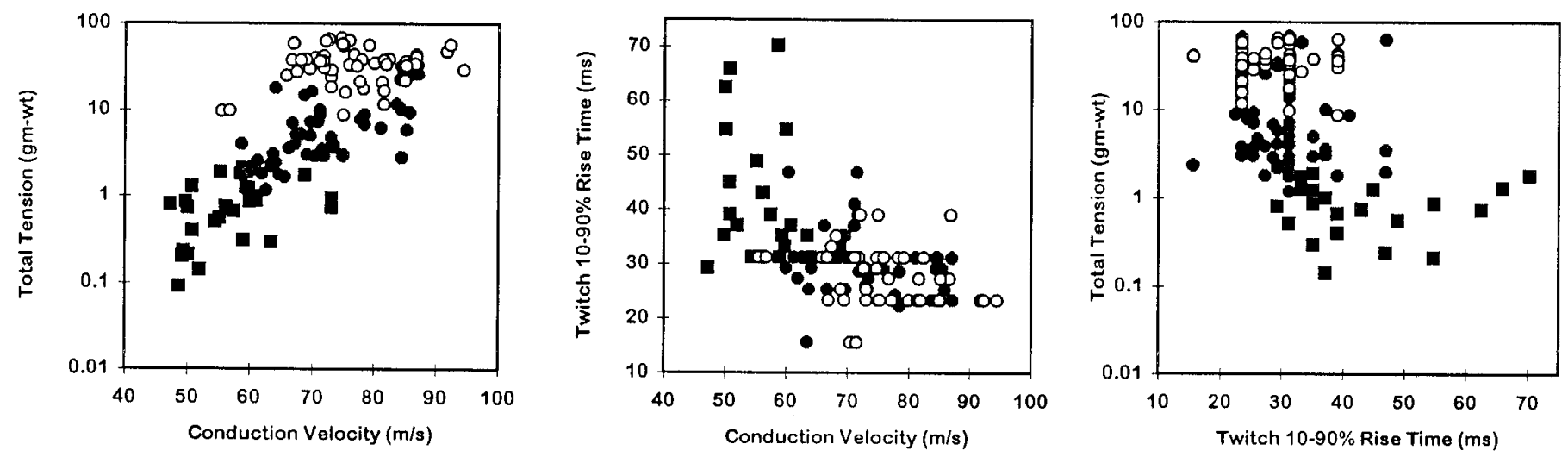

Figure 3. Contractile properties of EDLat MUs. Scatter plots are shown of total tetanic tension during a $150 \mathrm{~Hz}$ tetanus (logarithmic scale) versus conduction velocity $(A)$, twitch $10-90 \%$ rise time versus conduction velocity $(B)$, and total tension versus twitch rise time $(C)$. Significant correlations were found for all three relationships. Different symbols represent MUs of different physiological types: slow (ם), fast fatigue-resistant $(\boldsymbol{\bullet})$, or fast fatigable $(\bigcirc)$. Although the ranges of different MU types overlap considerably, significant differences related to MU type were found for all three variables.

some fraction of their tension on one or both of the other tendons. The degree to which this tension was exerted on one or on both of the other tendons can be judged by the degree to which a given point lies close to an edge of the triangle or symmetrically between two edges, respectively. Points midway along the edges, which would represent MUs that divided tension equally between two tendons while exerting none on the third, were notably absent. A few MUs exerted comparable tension on $\mathrm{t} 3$ and $\mathrm{t} 4$, and exerted a smaller fraction of their tension on 15 (Fig. 5, lower center). Few points fell near the center, indicating that few MUs exerted comparable tension on all 3 tendons.

To obtain quantitative indices of each MU's distribution of tension across the three tendons of EDLat, we calculated the OPI, DIV, and SEL for each EDLat MU as described in Materials and Methods. Histograms of OPI for types S, FR, and FF MUs and for all cat EDLat MUs together are shown in Figure $6 A$. All these histograms suggest a trimodal distribution, most evident for type FF and least evident for type S MUs. For the population as a whole, modal values of OPI were found at $+1.0,0.0$, and -0.5 . The OPI mode at +1.0 indicates that many units acted very selectively on $\mathrm{t} 3$. The mode at 0.0 indicates that many MUs exerted tension either preferentially on, or symmetrically about, $\mathrm{t} 4$. The mode at -0.5 indicates that many MUs exerting the greatest fraction of their tension on $\mathrm{t} 5$ also exerted appreciable tension on $\mathrm{t} 4$ or $\mathrm{t} 3$. The trimodal distribution of OPIs is consistent with three subpopulations of EDLat MUs, each subpopulation composed of MUs exerting the greatest fraction of their tension on one of the three tendons of the muscle, as evident in Figure 5. Table 1 gives descriptive statistics of OPI for type S, FR, and FF MUs. OPI showed no significant variation in relation to MU type, indicating that MUs of different types are not segregated in different regions of EDLat that act on different tendons.

Histograms of DIV for types S, FR, and FF MUs and for all cat EDLat MUs together are shown in Figure $6 \mathrm{~B}$. A wide range of DIV values was observed, especially for type S and FR MUs. Several EDLat MUs had DIV values between 1.0 and 1.5, indicating that they exerted less tension on the central tendon (t4) than on either the radial (t3) or ulnar (t5) tendon, a pattern not reported in EDC or ECU (Fritz et al., 1992). The DIV value of 0 , indicating complete selectivity for a single tendon, occurred artifactually for one type FR and eight type S MUs. For each of these MUs, two tendons were assigned a tension of 0 because their tension either was below the noise level, or showed unloading (see Materials and Methods). Table 1 gives descriptive statistics of DIV for type S, FR, and FF MUs. DIV varied significantly in relation to MU type. Although the distributions of DIV for type S versus type FR MUs did not differ significantly, the DIV distributions of type $S$ versus FF and of type FR versus FF did differ (KS tests, $p<$ $0.001)$. Their significantly lower DIV indices indicate that type FF MUs tended to be more highly selective for a given tendon in EDLat than type S or FR MUs, similar to the difference reported in the EDC of the cat (Fritz et al., 1992).

Figure $6 C$ shows histograms of SEL for types S, FR, and FF MUs and for all cat EDLat MUs together. Descriptive statistics are given in Table 1. The mean value of SEL for each MU type was greater than 0.5 , indicating that on average, MUs of each physiological type were relatively selective for one of the three tendons. As seen with the DIV, however, FF MUs were somewhat more selective (less divergent) than type S or FR MUs. SEL varied significantly in relation to MU type. Although the SEL distributions of type S versus type FR MUs did not differ significantly, the mean SEL of type $S$ versus FF, and of FR versus FF, did differ (KS tests, $p<0.001$ ). Type FF MUs in EDLat thus exerted tension more selectively on a single tendon than did type FR or S MUs.

\section{Monkey ED45 MUs}

We studied 57 ED45 MUs, from 10 to 24 MUs in individual monkeys. Because of conduction block, 34 MUs were stimulated in the radial nerve in the upper arm or forearm. Of the 23 MUs stimulated in the ventral roots, 1 was in spinal segment $\mathrm{C} 7,18$ were in $\mathrm{C} 8$, and 4 were in $\mathrm{T} 1$, consistent with segmental location of motoneuron columns for other macaque extrinsic finger muscles (Jenny and Inukai, 1983). Data from the one cynomologous monkey were similar to data from the three rhesus monkeys, and data from both species, therefore, were pooled for analysis. 
A

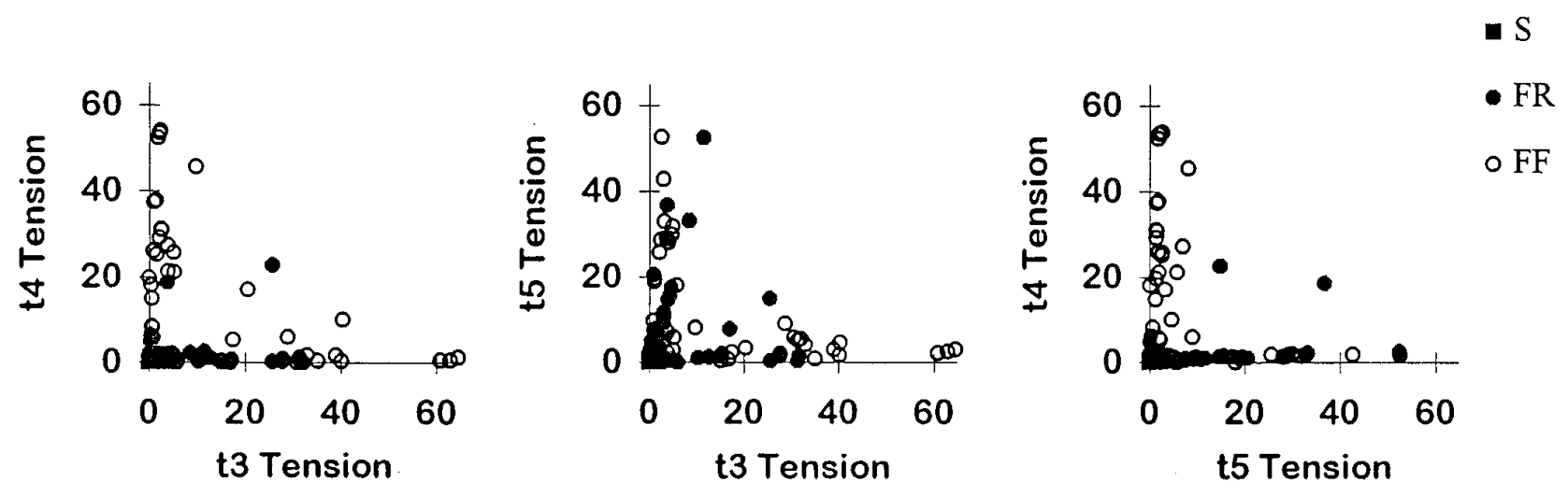

B
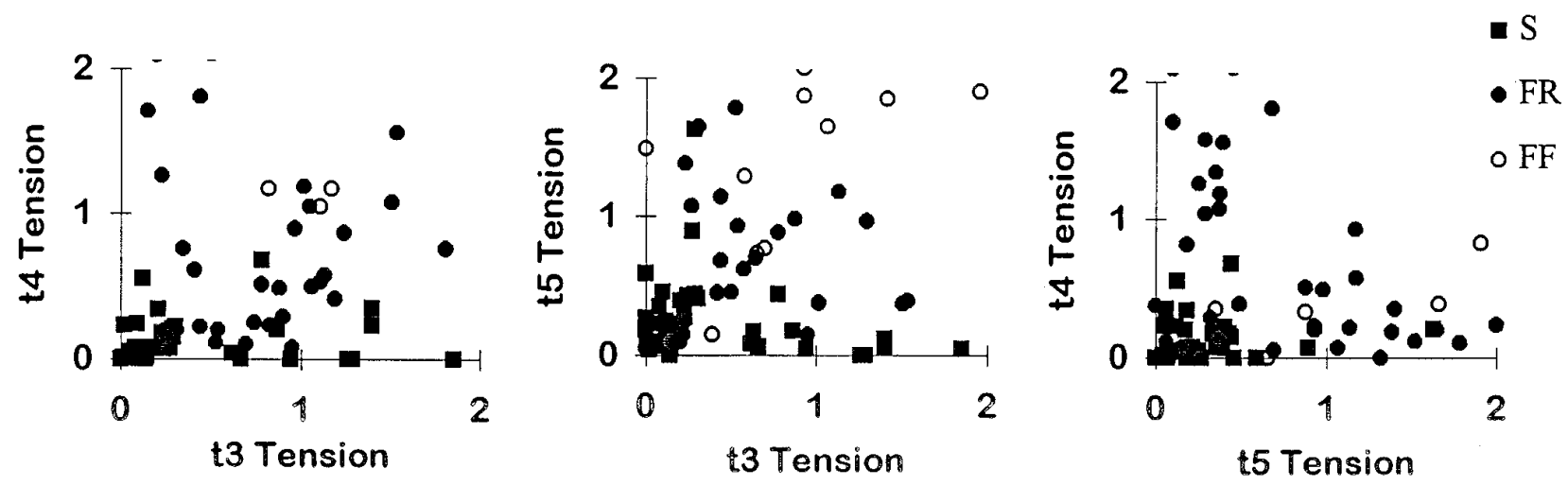

Figure 4. EDLat MU tension on two of three tendons. $A$, Scatter plots of the tension exerted by each MU on the tendon to digit $4(t 4)$ versus the tendon to digit $3(t 3 ;$ left $)$, on the tendon to digit $5(t 5)$ versus $\mathrm{t} 3$ (middle), and on $\mathrm{t} 4$ versus $\mathrm{t} 5$ (right). Most points in each plot lie close to one axis, indicating that the MU exerted tension relatively selectively on that tendon. $B$, Scatter plots of the same data at an expanded scale to show the data from small MUs. Symbols as in Figure 3.

Responses to tetanic stimulation at all three frequencies $(20,40$, and $150 \mathrm{~Hz}$ ) were recorded from 51 ED45 MUs. On the basis of comparison of the tension records of each MU, from $20 \mathrm{~Hz}$ and 40 $\mathrm{Hz}$ tetani, 2 were classified type S (4\%), 10 as FR (20\%), and 39 as $\mathrm{FF}(76 \%)$. Although we lack data on the innervation ratio of different physiological types in ED45, given that previous histochemical studies have shown ED45 to be composed of approximately $25 \%$ type I, $23 \%$ type IIa, and 52\% type IIb fibers (Maurer et al., 1995), type S and possibly type FR MUs were probably under-represented in our sample. We assume that underrepresentation of small MUs probably resulted from the technical difficulties encountered in working with the nerves of the monkey (see Materials and Methods), which may have preferentially impaired our ability to stimulate and to record action potentials from single small axons. Because our sample included only two type $\mathrm{S}$ MUs, we pooled type S and FR MUs into one fatigue-resistant group $(\mathrm{S}+\mathrm{FR})$ for subsequent analyses.

Table 2 gives descriptive statistics of the total maximum tetanic tension, conduction velocity, and twitch rise time for different MU types in ED45. Tetanic tension and twitch rise time each varied significantly in relation to MU type $(S+F R$ vs
FF, ANOVA; $p<0.0001)$. The difference in conduction velocity between the S+FR and FF MU groups, however, was less significant $(p<0.05)$. The distribution of ED45 conduction velocities was bimodal, with peaks at approximately 40 and 70 $\mathrm{m} / \mathrm{sec}$ and a trough at approximately $55 \mathrm{~m} / \mathrm{sec}$ (not illustrated, but see Fig. 7). A similar bimodal conduction velocity distribution was described for MUs of the EDC of the baboon (Eccles et al., 1968).

Figure 7 shows the inter-relationships of these properties of ED45 MUs. Considering all MU types together, a significant positive correlation was found between the logarithm of total tension and conduction velocity $(r=0.572 ; p<0.001$; Fig. $7 A)$, and highly significant negative correlations were found between twitch rise time and conduction velocity $(r=0.650 ; p<0.0001$; Fig. $7 B)$ and between total tension and twitch rise time $(r=$ $0.807 ; p<0.0001$; Fig. $7 C$ ). In contrast to the cat EDLat, separate relationships between the logarithm of total tension and conduction velocity for $\mathrm{S}+\mathrm{FR}$ versus FF MUs were not apparent in our data from the macaque ED45, which might have resulted from under-representation of type $S$ and FR MUs in our sample. 


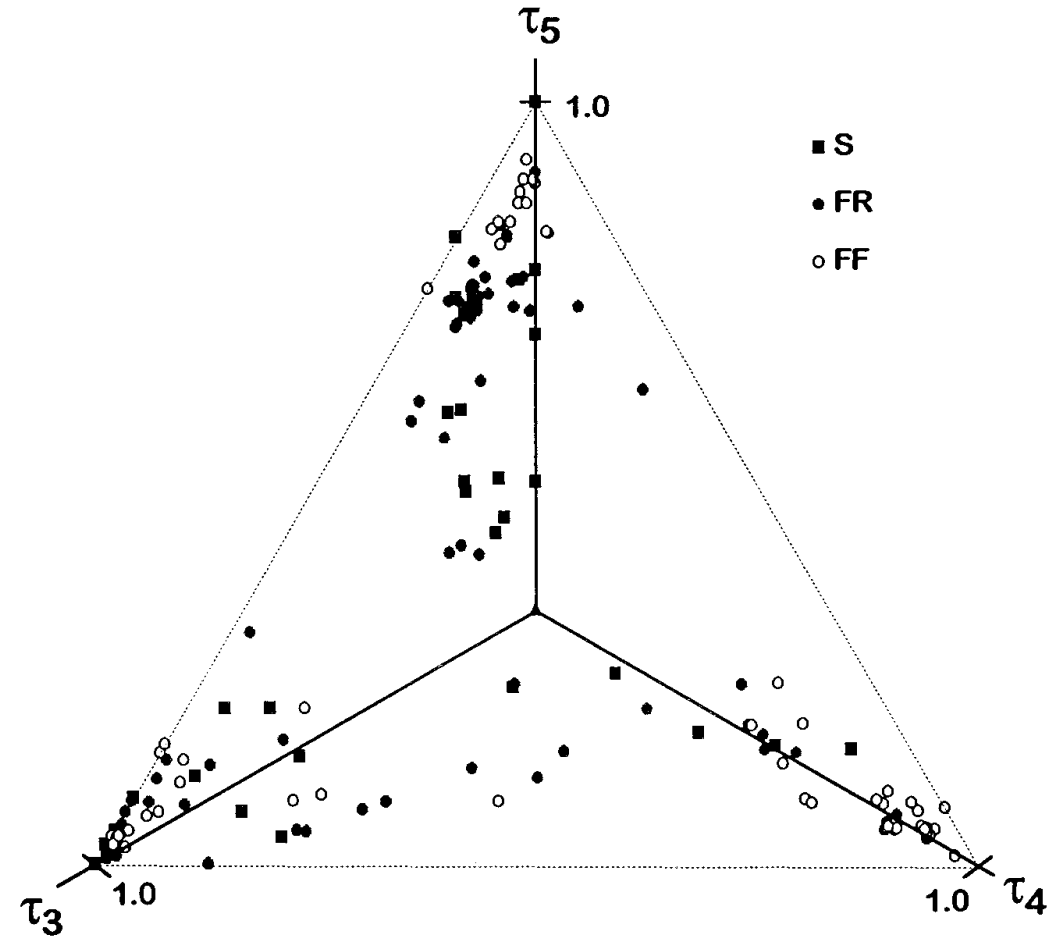

Figure 5. Fractional tension of EDLat MUs on all three tendons. Plotted in three dimensions, the fractional tensions of EDLat MUs all lie in the same plane $\left(\tau_{3}+\tau_{4}+\tau_{5}=1\right)$. The axes, therefore, have been rotated such that all points lie in the plane of the page. This plot reveals that rather than being scattered randomly over the possible area of an equilateral triangle (dashed lines), points representing most MUs lie close to one corner, indicating that they exerted tension preferentially on one tendon. Symbols as in Figure 3.

\section{Distribution of tension on the two tendons of ED45}

Figure 8 shows tension records from three ED45 MUs: one that produced tension rather selectively on $\mathrm{t} 4$ (Fig. $8 \mathrm{~A}$ ), another that produced comparable tension on $\mathrm{t} 4$ and $\mathrm{t} 5$ (Fig. $8 B$ ), and a third that produced tension rather selectively on t5 (Fig. $8 C$ ). Nonselective MUs like that shown in Figure $8 B$ were relatively uncommon in the EDLat of the cat, but constituted a significant fraction of the macaque ED45 sample. Figure $9 A$ shows a scatter plot of ED45 MU tension on $\mathrm{t} 4$ versus $\mathrm{t} 5$. To show the smaller MUs, the same data were replotted at an expanded scale in Figure $9 B$. Although many monkey ED45 MUs exerted tension rather selectively on either $\mathrm{t} 4$ or $\mathrm{t} 5$, and therefore are represented in these scatter plots as points lying close to either the 4 or $t 5$ axis, many other ED45 MUs exerted comparable tension on both $\mathrm{t} 4$ and $\mathrm{t5}$, with points representing these MUs widely scattered between the axes. Such an even scatter of points between the axes representing tension on different tendons was not seen in the data from cat EDLat MUs.

To compare MUs of different size, we normalized the tension of each MU in each tendon as the fraction of the total tension of that MU (see Materials and Methods, Eq. 1). In a two-dimensional scatter plot of fractional tensions, the points representing all ED45 MUs will lie on the line defined by $\tau_{4}+\tau_{5}=1$, because the sum of the fractional tensions for each MU is unity. (Again note that the subscripts used here denote the digit on which tension would be exerted via the tendon, whereas in the calculation of OPI and DIV the ordinal tendon numbers 1 and 2 must be used in place of digit numbers 4 and 5, respectively.) Such a plot is shown in Figure 9C. ED45 MUs are represented by points spread along this line. Many points lying near the midpoint of the line $\left(\tau_{4}\right.$ $\left.=\tau_{5}=0.5\right)$ represent MUs that exerted comparable tensions on $\mathrm{t} 4$ and $\mathrm{t} 5$. Although some points lie close to the intersection of the line with an axis $\left[\left(\tau_{4}=0, \tau_{5}=1\right)\right.$ or $\left.\left(\tau_{4}=1, \tau_{5}=0\right)\right]$, representing MUs that exerted tension selectively on just one tendon, points are not clustered close to these intersections. Compared to the
EDLat of the cat, the ED45 of the macaque thus seemed to have more MUs that distributed similar amounts of tension to both of the tendons of the muscle.

To quantify how ED45 MUs distributed their tension on the two tendons of the muscle, we calculated the OPI, DIV, and SEL indices for each MU. Histograms of OPI, DIV, and SEL for ED45 MUs are shown in Figure 10, and descriptive statistics are given in Table 2. Although the number of units is small, histograms of the OPI suggest that in addition to modes near +1 (all tension on $t 4$ ), 0 (tension divided evenly between $\mathrm{t} 4$ and $\mathrm{t} 5$ ), and -1 (all tension on t5), modes may be present at +0.5 and -0.5 , which would represent MUs that exerted roughly $75 \%$ of their tension on one tendon and $25 \%$ on the other. The distribution of DIV also shows that whereas some MUs exerted tension rather selectively on only one tendon (DIV near 0), the tension of many other MUs diverged evenly to the two tendons (DIV near 1). The SEL distributions also show that many MUs were highly selective for one tendon (SEL near 1), whereas others distributed tension evenly to the two tendons (SEL near 0), and still others put most of their tension on one tendon and some on the other (SELs between 0 and 1). Consistent with the OPI modes at -0.5 and +0.5 , the SEL distribution shows a mode at 0.5 , indicating that many ED45 MUs exerted about $75 \%$ of their tension on one tendon and $25 \%$ on the other.

In the EDLat of the cat, we found type FF MUs to be significantly more selective (higher SELs and lower DIVs) than type S or FR MUs, as was previously found for the EDC of the cat (Fritz et al., 1992). For the ED45 of the macaque, however, the histograms of DIV and SEL suggest a tendency for type FF MUs to be somewhat less selective (lower SELs and higher DIVs) than type $\mathrm{S}$ and FR MUs. Moreover, whereas OPIs near 0, indicating comparable tensions exerted on $\mathrm{t} 4$ and $\mathrm{t} 5$, were observed for several FF MUs in ED45, OPIs near 0 were not observed for type $\mathrm{S}$ and FR MUs. These trends, which may have resulted from 
A. OPI
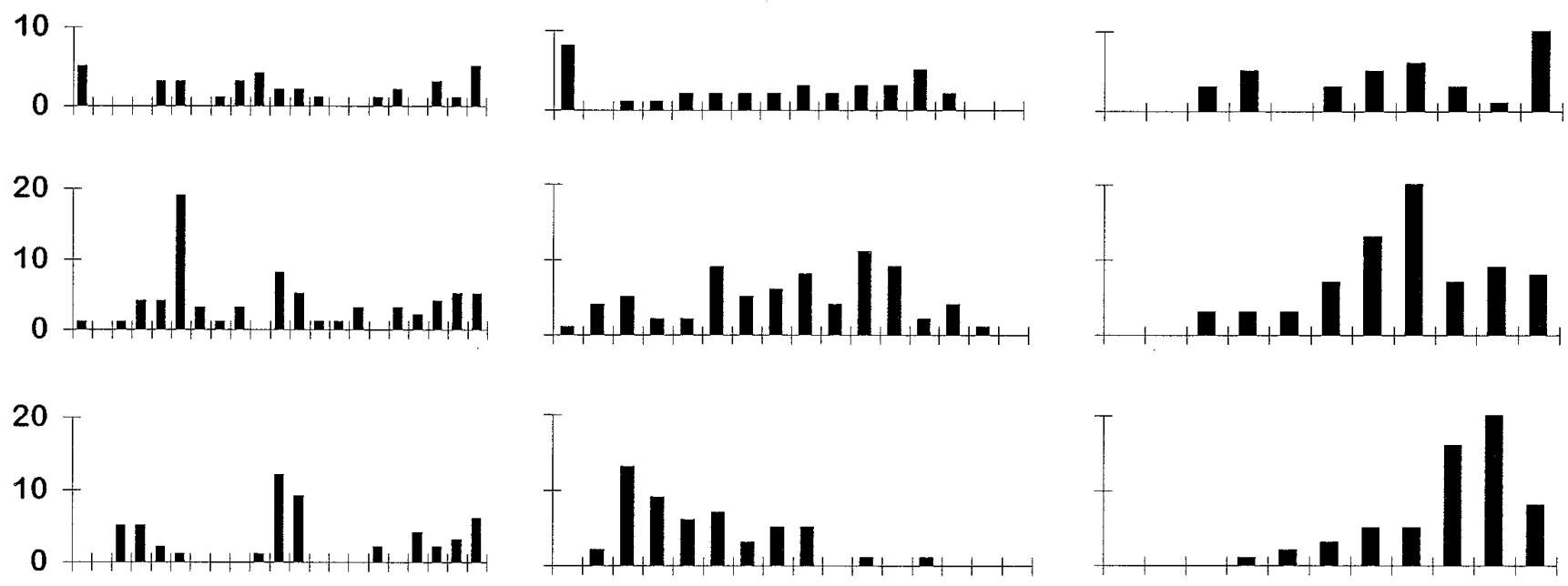

FF
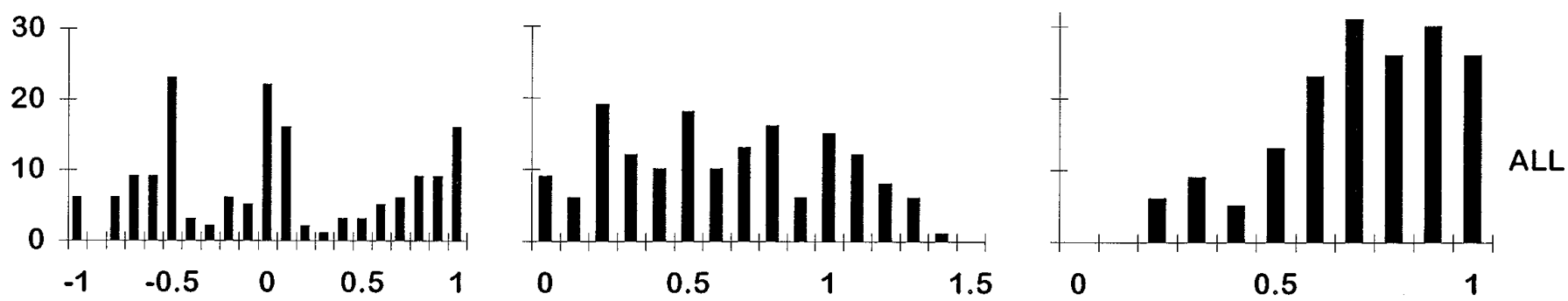

Figure 6. Histograms of OPI, DIV, and SEL indices for EDLat MUs. For each index, separate histograms are shown for EDLat MUs of each physiological type [slow $(S)$, fast fatigue resistant $(F R)$, and fast fatigable $(F F)$ ], as well as for all types together $(A L L)$. Horizontal axis labels at bottom indicate the upper limits of histogram bins; for example, the rightmost bin of SEL histograms, labeled 1.0, includes values from 0.901 to 1.000.

undersampling of type S and FR MUs, did not reach statistical significance for any of the three indices (KS tests).

Finally, we used OPI, DIV, and SEL values to compare the selectivity of monkey ED45 and cat EDLat MUs. For this purpose, SEL has the advantage of being both independent of the number of tendons a given muscle possesses, and independent of the particular tendons on which different fractions of the tension of a MU are exerted. No differences were found in OPI or DIV distributions between the monkey and cat muscles. But the SEL distributions did differ significantly between these two homologous muscles (KS test, $p<0.005$ ), confirming our observation that MUs in the ED45 of the macaque are less selective for a given tendon than are the MUs of the EDLat of the cat.

\section{DISCUSSION}

\section{MU properties}

Although extensively studied in many cat hindlimb muscles, MU physiology has been examined previously in only a few cat forelimb muscles: flexor carpi radialis, ECU, and EDC (Botterman et al., 1985; Fritz and Schmidt, 1992). The ranges and distributions of total maximum tetanic tension, axonal conduction velocity, and twitch rise time found here for the cat EDLat in general were similar to those reported for other forelimb muscles. These properties of cat EDLat MUs also resemble those reported for a number of hindlimb extrinsic digit muscles (flexor hallucis longus, flexor digitorum longus, and extensor digitorum longus) as well as for hindlimb muscles that act across the knee and ankle (Olson and Swett, 1966; Goslow, et al., 1972; Dum and Kennedy, 1980; Dum et al., 1982; Botterman et al., 1985; Emonet-Denand et al., 1988). The contractile and conduction properties of EDLat MUs thus fall within the ranges of other cat hindlimb and forelimb muscles.

In the ED45 of the monkey, twitch rise times were longer on average, and also ranged longer than those reported here for the EDLat of the cat, or those reported for other forelimb muscles, including the EDC of the baboon (Eccles et al., 1968). The ED45 of the monkey, therefore, may have relatively long contraction times, especially considering that slow twitch MUs may have been under-represented in our sample. The conduction velocities of ED45 MUs were distributed bimodally like those of the EDC of the baboon, in which the lower peak was at even slower velocities than in ED45 (approximately $30 \mathrm{~m} / \mathrm{sec}$; Eccles et al., 1968). The range of conduction velocities for ED45 MUs also extended lower than that of the EDLat of the cat, and conduction velocities of the EDC MUs of the baboon extended still lower $(20-25 \mathrm{~m} / \mathrm{sec})$. These observations raise the possibility that primate forelimb muscles have a subpopulation of relatively slowly conducting skeletomotor axons not found in most feline muscles.

The relationships between these properties of EDLat and ED45 MUs also resembled those reported for MUs in other muscles of the cat forelimb and hindlimb (Emonet-Denand et al., 1988). Significant positive correlation between the logarithm of total tension and conduction velocity, negative correlations between twitch rise time and conduction velocity, and negative 


\begin{tabular}{|c|c|c|c|}
\hline & $\mathrm{S}+\mathrm{FR}$ & $\mathrm{FF}$ & All \\
\hline$N$ & 12 & 39 & 51 \\
\hline Percentage & 23.5 & 76.5 & 100 \\
\hline \multicolumn{4}{|c|}{ Maximum tetanic tension (gm-wt) } \\
\hline Mean $\pm \mathrm{SD}$ & $1.48 \pm 0.82$ & $19.5 \pm 11.0$ & $15.2 \pm 12.3$ \\
\hline Range & $0.301-3.14$ & $3.25-45.2$ & $0.301-45.2$ \\
\hline$n$ & 12 & 39 & 51 \\
\hline \multicolumn{4}{|c|}{ Conduction velocity $(\mathrm{m} / \mathrm{sec})$} \\
\hline Mean $\pm \mathrm{SD}$ & $43.6 \pm 16.4$ & $55.1 \pm 13.1$ & $52.0 \pm 14.8$ \\
\hline Range & $28.1-71.2$ & $28.7-71.8$ & $28.1-71.8$ \\
\hline$n$ & 11 & 30 & 41 \\
\hline \multicolumn{4}{|c|}{ Twitch $10-90 \%$ rise time $(\mathrm{ms})$} \\
\hline Mean $\pm \mathrm{SD}$ & $65.5 \pm 17.4$ & $29.4 \pm 7.9$ & $37.9 \pm 18.8$ \\
\hline Range & $35.2-87.9$ & $10.1-48.8$ & $10.1-87.9$ \\
\hline$n$ & 12 & 39 & 51 \\
\hline \multicolumn{4}{|l|}{ OPI } \\
\hline Mean \pm SD & $-0.300 \pm 0.686$ & $-0.143 \pm 0.609$ & $-0.180 \pm 0.624$ \\
\hline Range & -0.911 to +1.0 & -0.960 to +0.967 & -0.960 to +1.0 \\
\hline$n$ & 12 & 39 & 51 \\
\hline \multicolumn{4}{|l|}{ DIV } \\
\hline Mean $\pm \mathrm{SD}$ & $0.478 \pm 0.299$ & $0.619 \pm 0.344$ & $0.586 \pm 0.337$ \\
\hline Range & $0-0.881$ & $0.064-1.0$ & $0-1.0$ \\
\hline$n$ & 12 & 39 & 51 \\
\hline \multicolumn{4}{|l|}{ SEL } \\
\hline Mean $\pm \mathrm{SD}$ & $0.692 \pm 0.217$ & $0.529 \pm 0.322$ & $0.567 \pm 0.307$ \\
\hline Range & $0.345-1.0$ & $0.001-0.967$ & $0.001-1.0$ \\
\hline$n$ & 12 & 39 & 51 \\
\hline
\end{tabular}

correlation between the logarithm of total tension and twitch rise time, all were found for cat EDLat MUs. Similar relationships were found for the macaque ED45. In these respects then, cat EDLat and macaque ED45 MUs were like MUs in any other muscle.

We did not collect data on the fatigue resistance of each MU, and therefore we have not determined whether the MU populations of the cat EDLat and macaque ED45 can be classified strictly into physiological types S, FR, and FF (Burke et al., 1973; Botterman et al., 1985; Fritz and Schmidt, 1992). Instead, assuming that both muscles contain all three types of MUs, we assigned each MU to one of these three physiological types by comparing the features of its tension responses during 20 and $40 \mathrm{~Hz}$ tetani. Our method of classifying the physiological type of each MU was similar to that of Petit et al. (1990), who found that this qualitative method resulted in few misclassifications when compared to classification based on more extensive examination, including a fatigue test. In this way, cat EDLat MUs in our sample were found to be $22.4 \%$ type $\mathrm{S}, 45.3 \% \mathrm{FR}$, and $32.3 \% \mathrm{FF}$, similar to the composition of other cat forelimb and hindlimb muscles (Olson and Swett, 1966; Goslow et al., 1972; Burke et al., 1973; Dum and Kennedy, 1980; Dum et al., 1982; Botterman et al., 1985; EmonetDenand et al., 1988; Fritz and Schmidt, 1992). Misclassification of a small number of MUs might have made these percentages slightly inaccurate. In our sample of macaque ED45 MUs, 4\% were type S, $20 \% \mathrm{FR}$, and $76 \% \mathrm{FF}$. As described in Results, however, this sample probably under-represented types S and FR chiefly because of the technical difficulties we experienced in stimulating single MUs in the nerve of the monkey. In neither the cat nor monkey MU sample, however, would misclassification of the physiological type of a small fraction of the MUs have compromised our conclusions concerning tension distribution.

\section{Distribution of MU tension to the tendons of multitendoned muscles}

Although MUs in the EDLat of the cat and the ED45 of the macaque have many properties that closely resemble MUs in monotendoned muscles, EDLat and ED45 MUs exert tension on multiple, independent insertion tendons. We examined how individual EDLat MUs distributed their tension among the three tendons of the muscle in part to determine whether EDLat might contain separate functional subdivisions serving each tendon. If every MU in a muscle produces comparable tension on all the tendons of the muscle, then subsets of its MU pool would be unable to act preferentially on a given finger. But if each MU produces tension mainly on one tendon, then activation of only those MUs that act on a particular tendon could exert tension preferentially on that tendon, and the muscle might be functionally subdivided. Each subdivision would provide a different distribution of tension on the tendons, acting preferentially on a different tendon. Previous studies of tension distribution by MUs in the EDC and ECU of the cat indicated that most MUs in these muscles produced tension that diverged to more than one insertion tendon, and that the center of effort (OPI) was distributed evenly over the range from the most radial to most ulnar insertion (Fritz et al., 1992). These features suggested that, in cats, neither EDC or ECU are likely to consist of functional subdivisions serving different tendons.

Most EDLat MUs, however, were found here to exert tension rather selectively on one of the three tendons of the muscle. Few EDLat MUs distributed their tension evenly to two or to all three tendons. For the population of EDLat MUs, the OPI characterizing the radial-to-ulnar center of effort had a trimodal distribution, consistent with three subpopulations of MUs that focus tension preferentially on different tendons. This contrasts with the continuous distributions of OPI described for EDC and ECU (Fritz et al., 1992). Furthermore, DIV, an index of the degree to which MU tension diverges across tendons, reached lower values $(<0.3)$ in many EDLat MUs than the lowest values reported for any EDC or ECU MU, indicating that tension from many EDLat MUs is more selectively focused on one tendon. Finally, SEL, an index of the degree to which a MU is selective for one tendon independent of the radial-to-ulnar tendon order, was skewed for EDLat MUs toward values greater than 0.5, again indicating that most EDLat MUs exert tension relatively selectively on one tendon. This selectivity raises the possibility that EDLat might be organized into three separate functional subdivisions, each acting preferentially on the tendon to digit 3,4 , or 5 .

Because the vast majority of EDLat MUs exerted measurable tension on all three tendons, however, no functional subdivision could act on a single tendon with complete selectivity. Spread of tension to other tendons might occur for two reasons. First, tension might spread to other tendons passively via epimysial connective tissue, or by lateral compression of passive muscle fibers due to thickening of fibers that shorten actively (Loeb et al., 1987; Fritz et al., 1992). Second, tension might be exerted on other tendons actively. Although most muscle fibers of a given MU 

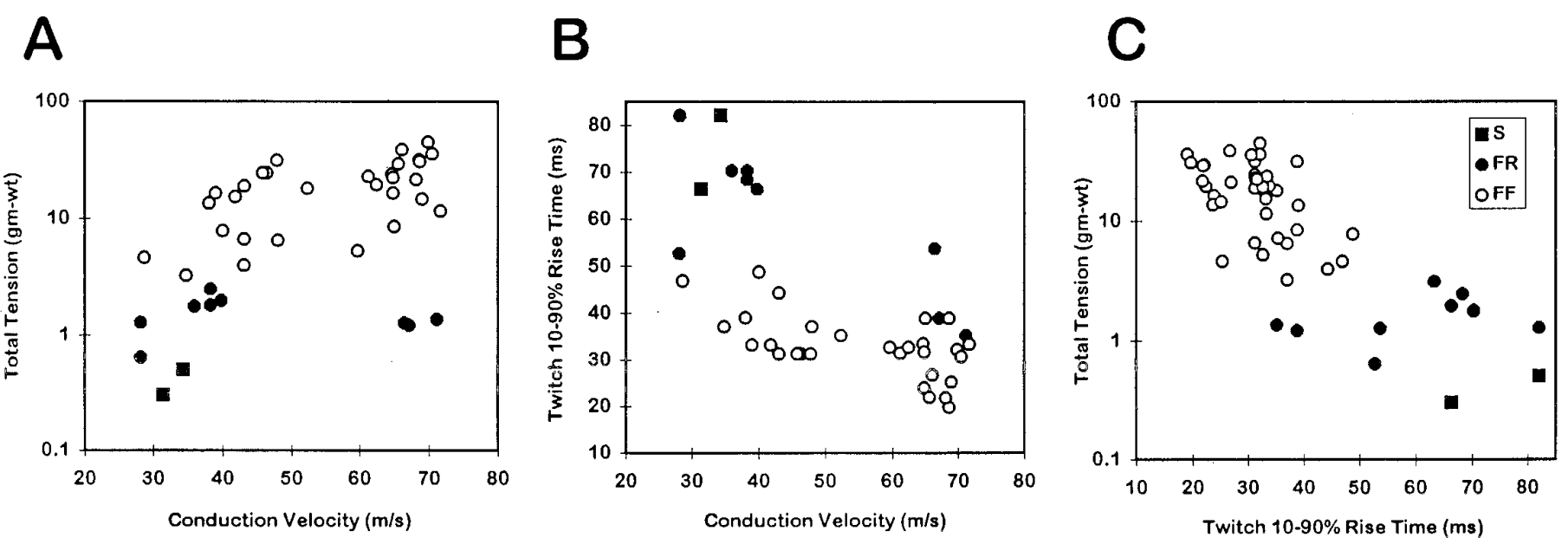

Figure 7. Contractile properties of macaque ED45 MUs. Scatter plots are shown of total tension during a $150 \mathrm{~Hz}$ tetanus (logarithmic scale) versus conduction velocity $(A)$, twitch $10-90 \%$ rise time versus conduction velocity $(B)$, and total tension versus twitch rise time $(C)$. Significant correlations were found for all three relationships. Symbols as in Figure 3.

Tw $\quad 20 \mathrm{~Hz}$

A
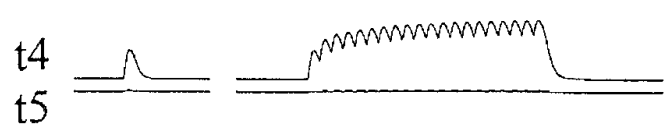

B

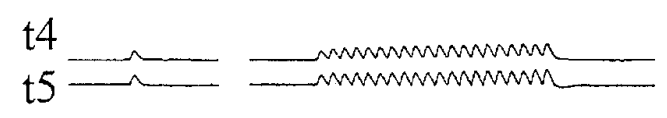

$\mathrm{C}$

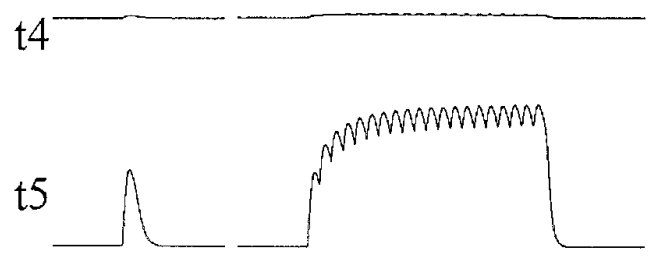

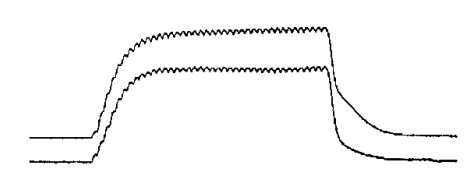

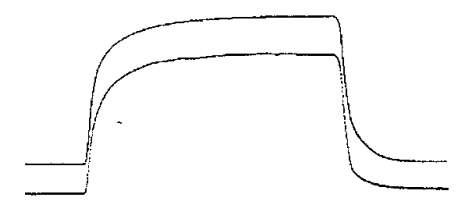

$40 \mathrm{~Hz}$
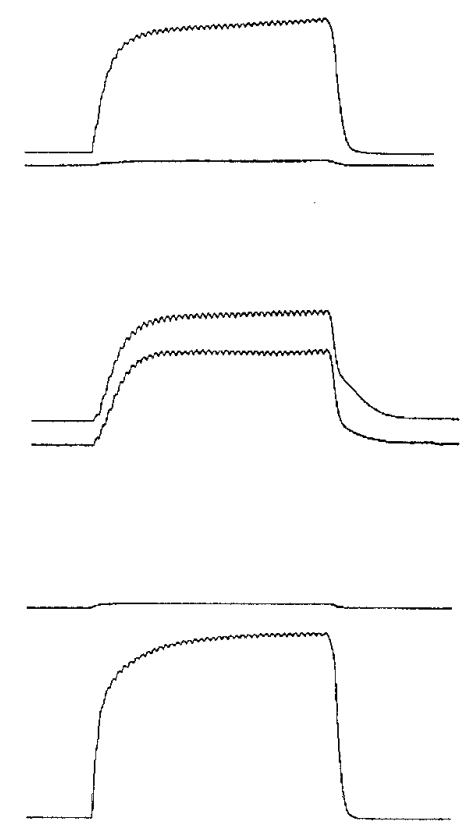

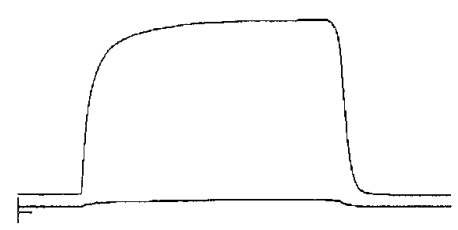

$150 \mathrm{~Hz}$

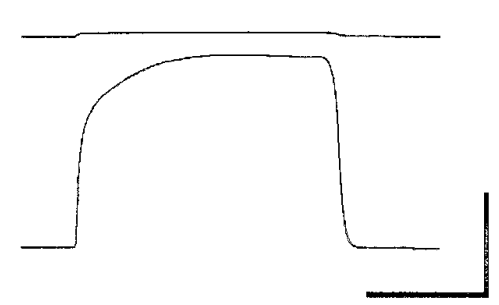

Figure 8. Three monkey ED45 MUs. For each MU, simultaneous records of the tension on the tendon to digit $4(t 4)$ and on the tendon to digit 5 ( $t 5)$ are shown during a single twitch and during tetanic stimulation at 20,40 , and $150 \mathrm{~Hz}$. $A$, The MU shown exerted tension selectively on t4 (OPI $=0.923$; $\mathrm{DIV}=0.149 ; \mathrm{SEL}=0.923) ; B$, the MU exerted equivalent tension on both $\mathrm{t} 4$ and $\mathrm{t} 5$ (OPI $=0.031 ; \mathrm{DIV}=0.999 ; \mathrm{SEL}=0.031) ; C$, the MU exerted tension selectively on t5 (OPI $=-0.960 ; \mathrm{DIV}=0.078 ; \mathrm{SEL}=0.960)$. Horizontal calibration bar $=0.5$ sec; vertical calibration bar $=12.5$ gm-wt in $A$ and $C, 5$ gm-wt in $B$.

might insert on one tendon, a minority of muscle fibers of the same MU might insert on other tendons. Some muscle fibers of a given MU would then be scattered over much of the entire muscle belly (Burke and Tsairis, 1973; Loeb et al., 1987). In this case, the functional subdivisions of the muscle would not correspond strictly to neuromuscular compartments, but each subdivision nevertheless would be capable of acting preferentially on one of the tendons of the muscle if the corresponding subpool of motoneurons could be selectively activated (Chanaud et al., 1991; Burke, 1995).

In contrast to the EDLat of the cat, the ED45 of the monkey contained many MUs that exerted comparable tensions on the two tendons of the muscle. Whereas the least selective cat EDLat MUs were among the smallest, the least selective monkey ED45 


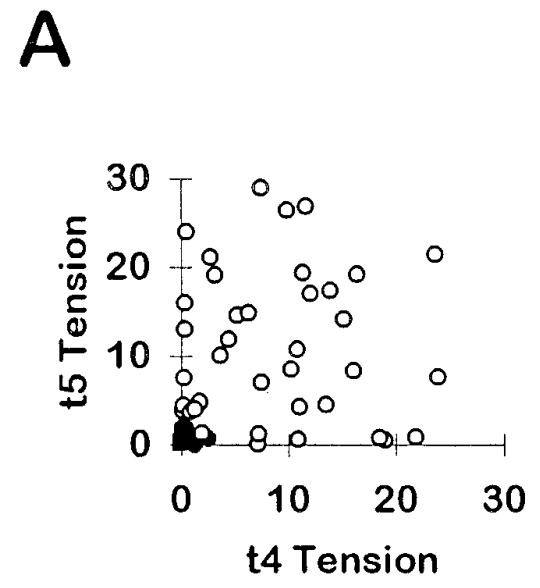

B

C
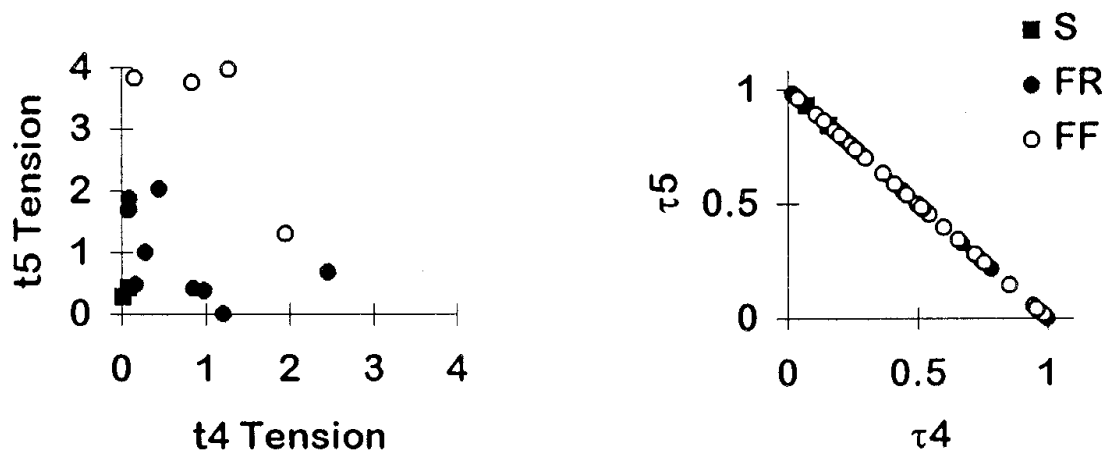

Figure 9. Scatter plots of ED45 MU tension. $A$, Scatter plots of the tension exerted by each MU on the tendon to digit $4(t 4)$ versus the tendon to digit $5(t 5)$. Although many points in each plot lie close to one axis, indicating that those MUs exerted tension relatively selectively on that tendon, many other points lie well between the axes, indicating that those MUs exerted comparable tension on both $\mathrm{t} 4$ and $\mathrm{t} 5$. $B$, Scatter plots of the same data at an expanded scale to show the data from small MUs. $C$, Plotted in two dimensions, the fractional tensions of ED45 MUs all lie on the same line $\left(\tau_{4}+\tau_{5}=1\right)$. Some points lie near one of the axes, and therefore represent MUs that exerted tension rather selectively on that tendon. But many points are spread along the line, indicating that many MUs exerted comparable fractions of their tension on both 44 and $t 5$. Symbols as in Figure 3.

\section{A. OPI}
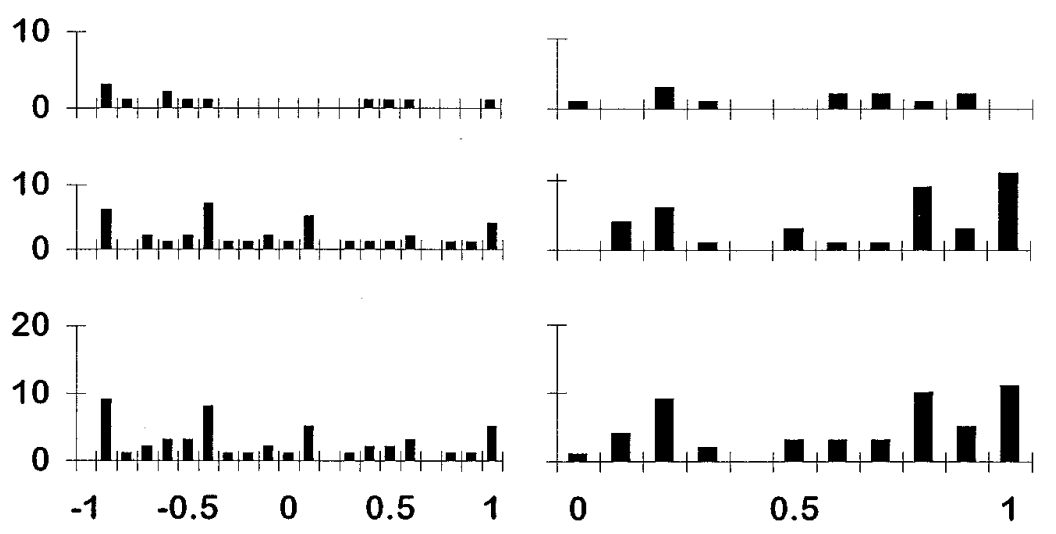

\section{SEL}

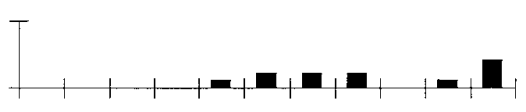

FR+S

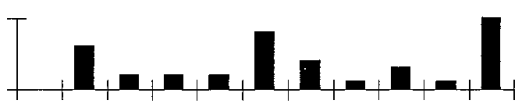

FF

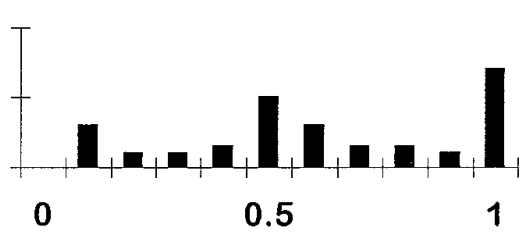

Figure 10. Histograms of OPI, DIV, and SEL indices for ED45 MUs. For each index, separate histograms are shown for slow plus fast fatigue-resistant MUs $(S+F R)$, for fast fatigable MUs $(F F)$, and for all types together $(A L L)$. Labels on the horizontal axes at bottom indicate the upper limit of each histogram bin.

MUs were among the largest. This difference raises the possibility that the larger, nonselective MUs in ED45 were in fact two simultaneously stimulated MUs, one selective for $\mathrm{t} 4$, the other for $\mathrm{t} 5$, especially given that in two monkeys we used stimulation of the radial nerve when stimulation of ventral rootlets failed to activate MUs. MU stimulation in the ventral rootlets has two advantages. First, because axons of the MUs to a given muscle are dispersed over multiple rootlets, simultaneous stimulation of two MUs in the muscle under study is less common. Second, because the conduction distance from the ventral rootlets to the muscle nerve is relatively long, simultaneous stimulation of two MUs is more likely to be recognized by recording two temporally separate axonal action potentials in the muscle nerve. In contrast, stimulation in the peripheral nerve has the disadvantages that the axons to a given muscle are bundled together, and the short conduction distance decreases the temporal dispersion of axonal action potentials. Nevertheless, we are reasonably confident that most of the nonselective ED45 MUs we recorded were in fact single MUs for two reasons. First, we carefully adjusted the stimulation for each MU to be just above threshold during all stimuli-single twitches as well as tetani at 20,40, and $150 \mathrm{~Hz}$, excluding instances in which the MU under study failed to contract consistently, or in which the contraction of a second MU appeared intermittently. This process minimized the likelihood of simultaneously recording the tension of two MUs with similar thresholds and conduction velocities, whether stimulating in the ventral rootlets or in the radial nerve. Second, equivalent proportions of nonselective MUs were recorded in all four monkeys, those in which MUs were stimulated in the ventral rootlets as well as those in which MUs were stimulated in the radial nerve. We therefore conclude that ED45 contains a significant fraction of relatively large, nonselective MUs that exert comparable tension on both $\mathrm{t} 4$ and $\mathrm{t} 5$.

The presence of such nonselective MUs in ED45 would be consistent with two previous observations. First, ED45 is innervated by two nerve branches, one innervating distal muscle fascicles that act on $\mathrm{t} 4$, and the other innervating some proximal 
muscle fascicles that act on $\mathrm{t} 4$ plus all the fascicles acting on $\mathrm{t} 5$ (Serlin and Schieber, 1993). Second, electromyographic recordings failed to identify regions in ED45 that were differentially activated during individuated finger movements (Schieber, 1995). Although not excluding the possibility that the distal neuromuscular compartment in ED45 acts only on $\mathrm{t} 4$ and the proximal compartment acts on both $\mathrm{t} 4$ and $\mathrm{t} 5$, these observations on $\mathrm{MU}$ biomechanics, neuromuscular anatomy, and electromyography combine to make it unlikely that the ED45 of the monkey has separate functional subdivisions that simply serve either $\mathrm{t} 4$ or $\mathrm{t} 5$. In this regard it is noteworthy that the human homolog, extensor digiti minimi (EDM), affords more selective action not by having divided into two separate compartments or muscles for $\mathrm{t} 4$ and $\mathrm{t} 5$, but instead by having lost its tendon to digit 4 , such that the muscle acts only on digit 5 . The spectrum across species (from cat to monkey to human) thus suggests that although preferential action on one tendon in a multitendoned muscle might be achieved by functional subdivision (cat EDLat), an evolutionary mechanism for producing even greater selectivity might involve limiting the action of the muscle to fewer (monkey ED45) or to only a single (human EDM) tendon.

\section{REFERENCES}

Balice-Gordon RJ, Thompson WJ (1988) The organization and development of compartmentalized innervation in rat extensor digitorum longus muscle. J Physiol 398:211-231.

Botterman BR, Iwamoto GA, Gonyea WJ (1985) Classification of motor units in flexor carpi radialis muscle of the cat. J Neurophysiol 54:676-690.

Burke RE, Levine DN, Tsairis P, Zajac III FE (1973) Physiological types and histochemical profiles in motor units of the cat gastrocnemius. J Physiol (Lond) 234:723-748.

Burke RE (1995) Selective recruitment of motor units. In: Motor control: concepts and issues (Humphrey DR, Freund H-J, eds), pp 5-21. New York: Wiley.

Burke RE, Tsairis P (1973) Anatomy and innervation ratios in motor units of cat gastrocnemius. J Physiol (Lond) 234:749-765.

Chanaud CM, Pratt CA, Loeb GE (1991) Functionally complex muscles of the cat hindlimb. V. The roles of histochemical fiber-type regionalization and mechanical heterogeneity in differential muscle activation. Exp Brain Res 85:300-313.

Drake III W, Li Y, Rothschild MA, Wu BL, Biller HF, Sanders I (1993) A technique for displaying the entire nerve branching pattern of a whole muscle: results in 10 canine posterior cricoarytenoid muscles. Laryngoscope 103:141-148.

Dum RP, Kennedy TT (1980) Physiological and histochemical characteristics of motor units in cat tibialis anterior and extensor digitorum longus muscles. J Neurophysiol 43:1615-1630.

Dum RP, Burke RE, O’Donovan MJ, Toop J, Hodgson JA (1982) Motor-unit organization in flexor digitorum longus muscle of the cat. J Neurophysiol 47:1108-1125.

Eccles RM, Phillips CG, Chien-Ping W (1968) Motor innervation, motor unit organization and afferent innervation of $\mathrm{m}$. extensor digitorum communis of the baboon's forearm. J Physiol (Lond) 198:179-192.

Emonet-Denand F, Hunt CC, Petit J, Pollin B (1988) Proportion of fatigue-resistant motor units in hindlimb muscles of cat and their relation to axonal conduction velocity. J Physiol (Lond) 400:135-158.

English AW (1984) An electromyographic analysis of compartments in cat lateral gastrocnemius muscle during unrestrained locomotion. J Neurophysiol 52:114-125.

English AW (1990) Development of compartmentalized innervation of the rat gluteus maximus muscle. J Comp Neurol 301:104-113.

English AW, Letbetter WD (1982) Anatomy and innervation patterns of cat lateral gastrocnemius and plantaris muscles. Am J Anat 164:67-77.

English AW, Weeks OI (1984) Compartmentalization of single muscle units in cat lateral gastrocnemius. Exp Brain Res 56:361-368.

English AW, Weeks OI (1987) An anatomical and functional analysis of cat biceps femoris and semitendinosus muscles. J Morphol 191:161-175.
English AW, Wolf SL, Segal RL (1993) Compartmentalization of muscles and their motor nuclei: the partitioning hypothesis. Phys Ther 73:857-867.

Fritz N, Schmidt C (1992) Contractile properties of single motor units in two multi-tendoned muscles of the cat distal forelimb. Exp Brain Res 88:401-410.

Fritz N, Illert M, Saggau P (1986) Location of motoneurones projecting to the cat distal forelimb. I. Deep radial motornuclei. J Comp Neurol 244:286-301.

Fritz N, Schmidt C, Yamaguchi T (1992) Biomechanical organization of single motor units in two multi-tendoned muscles of the cat distal forelimb. Exp Brain Res 88:411-421.

Gorska T, Sybirska E (1980) Effects of pyramidal lesions on forelimb movements in the cat. Acta Neurobiol Exp 40:843-859.

Goslow GE, Jr, Stauffer EK, Nemeth WC, Stuart DG (1972) Digit flexor muscles in the cat: their action and motor units. J Morphol 137:335-342.

Herring SW, Grimm AF, Grimm BR (1979) Functional heterogeneity in a multipinnate muscle. Am J Anat 154:563-576.

Jenny AB, Inukai J (1983) Principles of motor organization of the monkey cervical spinal cord. J Neurosci 3:567-575.

Kilbreath SL, Gandevia SC (1994) Limited independent flexion of the thumb and fingers in human subjects. J Physiol (Lond) 479:487-497.

Loeb GE, Pratt CA, Chanaud CM, Richmond FJ (1987) Distribution and innervation of short, interdigitated muscle fibers in parallel-fibered muscles of the cat hindlimb. J Morphol 191:1-15.

Maurer JJ, Singer MA, Schieber MH (1995) Fiber type composition of morphologic regions in the macaque multitendoned finger muscles. Acta Anat 154:216-223.

Olson CB, Swett Jr CP (1966) A functional and histochemical characterization of motor units in a heterogeneous muscle (flexor digitorum longus) of the cat. J Comp Neurol 128:475-498.

Pare EB, Stern JT, Schwartz JM (1981) Functional differentiation within the tensor fascia latae. J Bone Joint Surg 63A:1457-1471.

Petit J, Filippi GM, Emonet-Denand F, Hunt CC, Laporte Y (1990) Changes in muscle stiffness produced by motor units of different types in peroneus longus muscle of cat. J Neurophysiol 63:190-197.

Pratt CA, Loeb GE (1991) Functionally complex muscles of the cat hindlimb. I. Patterns of activation across sartorius. Exp Brain Res 85:243-256.

Pratt CA, Chanaud CM, Loeb GE (1991) Functionally complex muscles of the cat hindlimb. IV. Intramuscular distribution of movement command signals and cutaneous reflexes in broad, bifunctional thigh muscles. Exp Brain Res 85:281-299.

Sanders I, Wu BL, Mu L, Biller HF (1994) The innervation of the human posterior cricoarytenoid muscle: evidence for at least two neuromuscular compartments. Laryngoscope 104:880-884.

Schieber MH (1991) Individuated finger movements of rhesus monkeys: a means of quantifying the independence of the digits. J Neurophysiol 65:1381-1391.

Schieber MH (1993) Electromyographic evidence of two functional subdivisions in the rhesus monkey's flexor digitorum profundus. Exp Brain Res 95:251-260.

Schieber MH (1995) Muscular production of individuated finger movements: the roles of extrinsic finger muscles. J Neurosci 15:284-297.

Schmied A, Ivarsson C, Fetz EE (1993) Short-term synchronization of motor units in human extensor digitorum communis muscle: relation to contractile properties and voluntary control. Exp Brain Res 97:159-172.

Segal RL, Wolf SL, DeCamp MJ, Chopp MT, English AW (1991) Anatomical partitioning of three multiarticular human muscles. Acta Anat 142:261-266.

Serlin DM, Schieber MH (1993) Morphologic regions of the multitendoned extrinsic finger muscles in the monkey forearm. Acta Anat 146:255-266.

Soechting JF, Flanders M (1992) Organization of sequential typing movements. J Neurophysiol 67:1275-1290.

Vanden Noven S, Gardiner PF, Seburn KL (1994) Motoneurons innervating two regions of rat medial gastrocnemius muscle with differing contractile properties. Acta Anat 150:282-293.

Whishaw IQ, Gorny B (1994) Arpeggio and fractionated digit movements used in prehension by rats. Behav Brain Res 60:15-24.

Windhorst U, Hamm TM, Stuart DG (1989) On the function of muscle and reflex partitioning. Behav Brain Sci 12:629-645. 